\title{
"But One Must Not Legalize the Mentioned Sin": Phenomenological vs. Dynamical Treatments of Rods and Clocks in Einstein's Thought
}

\author{
Marco Giovanelli \\ marco.giovanelli@uni-tubingen.de
}

\begin{abstract}
This paper offers a historical overview of Einstein's vacillating attitude towards 'phenomenological' and 'dynamical' treatments of rods and clocks in relativity theory. In Einstein's view, a realistic microscopic model of rods and clocks was needed to account for the very existence of measuring devices of identical construction that always measure the same unit of time and the same unit of length. It will be shown that the empirical meaningfulness of both relativity theories depends on what, following Max Born, one might call the 'principle of the physical identity of the units of measure'. In an attempt to justify the validity of such a principle, Einstein was forced by different interlocutors, in particular Hermann Weyl and Wolfgang Pauli, to deal with the genuine epistemological, rather then physical question of whether a theory should be required to describe the material devices needed for its own verification.
\end{abstract}

Keywords: Albert Einstein, Relativity Theory, Rods and Clocks, Hermann Weyl, Wolfgang Pauli, Constructive Relativity, Confirmational Holism

Intrinsically Brobdingnag and
Lilliput are precisely the same; it
needs an intruding Gulliver-an
extraneous standard of length- - to
make them appear different.

A. S. Eddington

\section{Introduction}

In recent years, thanks mainly to Harvey Brown's seminal work (for the most part collected in Brown, 2005), increasing attention has been drawn to Einstein's 'self-confessed sin' of treating rods and clocks as 'primitive', 'self-sustained', or 'unstructured' entities. Brown rightly insisted that Einstein was aware that rods and clocks are actually 'structured dynamical entities', 'composite bodies', etc. the behavior of which depends on the structural properties of the forces responsible for the microstructure of matter. Therefore-as Einstein put it paradigmatically in his self-written 'obituary' in a volume for the Library of Living Philosophers edited by Paul Arthur Schilpp — the 'sin' of treating rods and clocks as ultimate elements incapable of further explication should not be "legalized" (Einstein, 1949a, 59). It was a temporary expedient, necessary only because physics at that time did not posses the adequate conceptual tools to construct rods and clocks explicitly from the fundamental physical quantities in the theory, from fields alone, or in the worst case scenario, from fields and particles together (Barbour, 2007, 587).

Einstein's vacillating attitude towards the role, indispensable or provisional, of rods and clocks in both of his theories, has usually been cast in the well-known opposition between constructive and principle theories (Einstein, 1919b; see Howard, 2005, for more details). In Einstein's original 'principle strategy', the behavior of rods and clocks was deduced from the postulates of the theory without introducing a realistic microscopic model of their material constitution. However, if a suitable "constructive" theory of matter was eventually at hand, rods and clocks would be thought of as rather complicated physical systems obeying fundamental dynamical laws. The ambition of this paper is not to solve the vexed question of the "arrow of explanation in special relativity" (Gorski, 2010), namely, whether it is the behavior of rods and clocks that explains the symmetries of space-time (Brown and Pooley, 2006), or if it is the other way around (cf. e.g. Norton, 2008; Janssen, 2009). Rather, it attempts to take a cue from this debate in order to offer an overall historical survey of Einstein's concerns about a tension between 'phenomenological' and 'dynamical' treatments of rods and clocks. This paper will therefore follow as closely as possible the historical development (or rather, as we shall 
see, the surprising constancy) of Einstein's stance towards this issue in published and unpublished writings.

In particular, this paper will document the context in which Einstein, probably at the beginning of 1917, seems to have first expressed, in his private correspondence, concerns about the use of complicated material systems as measuring devices (section 2). It will consider the circumstances in which Einstein, in the 1920s, when pressed by the epistemological objections raised by different interlocutors - in particular Hermann Weyl (Scholz, 2007) and the young Wolfgang Pauli (Stachel, 2005) - felt the urgency to articulate his point of view in public writings (section 3). Thereby, he outlined a two-stage epistemological strategy, vacillating between a provisional and principled attitude toward the use of rods and clocks in relativity theory (section 4), to which he remained faithful until the end of his life (section 5). Even if other interlocutors are worthy of consideration (in particular Arthur Stanley Eddington), I have decided to concentrate on the German debate, which for obvious reasons offers a particularly rich dialogical network comprised of private correspondences, public confrontations, and philosophical disputes (for the dialogical approach to the history of science, cf. Beller, 1999).

Some of this material has been analyzed in some excellent historical and philosophical literature (Stachel, 1989; Howard, 1994; Ryckman, 2005; Howard, 2007; Fogel, 2008; and Howard, 2014, to mention a few). However, as far I can see, there is no overall historical-critical overview of Einstein's stance towards this issue over the years. This is unfortunate, since the question of whether the behavior of measuring devices should be derived from the theory equations and not stipulated independently has often been discussed in the recent philosophical debate by appealing to a limited group of passages written by Einstein on disparate occasions.

As I will try to show, if inserted into the proper historical setting, Einstein's dissatisfaction about his initial operational treatment of rods and clocks, and his corresponding appeal to a dynamical explanation, does not seem to ever actually address the question in which the contemporary debate is most interested: whether space-time lives a 'parasitic' existence at the expense of the dynamical phenomena of the contraction of rods and the dilation of clocks, or whether in a space-time formulation of special relativity rods and clocks need not to appear at all. In particular, there is no textual evidence that Einstein ever argued in favor of a dynamical explanation of rods and clocks in the context of his opposition between constructive and principle theories, so that constructive relativity would finally explain dynamically what relativity as a principle theory had described only kinematically.

It is undeniable that early on, Einstein expressed the conviction that a dynamical account of rods and clocks was needed in both special and general relativity. However, this was not meant to explain rod contractions and clock dilations (cf. Janssen 2009, 39). What needed to be explained dynamically was precisely the opposite fact - that, to put it somewhat provocatively, there exist clocks that do not slow down and rods that do not shorten in any circumstances. More precisely, in both special and general relativity, it is more or less tacitly assumed that identically constructed rods and identically constructed clocks, which measure respectively the same length and show the same rate of ticking when they are held side by side at one location at a certain time, will always agree on their readings if carefully placed next to each other while at rest again, whatever their intermediate histories may have been. In other words, a dynamical explanation should account for the very existence of good rods and clocks of identical construction that always measure the same true proper time and proper length, whatever may have happened to them in the past (Valente, 2013).

Relativity theory does not provide any clues for assuming that physical systems with these characteristics really exist in nature, nor does it tell us how to recognize and identify them if they did. Rather, one must proceed to a tentative and provisional identification. For instance, experience furnishes sufficient reasons to regard two atoms of the same substance as identical clocks. After it has been 'stipulated' that atoms could play the role of good clocks (i.e., that the frequency of an atom is always its proper frequency), then it is expected that these systems should behave according to the predictions of the theory. In this sense the theory is said to be empirically testable. In particular, if we compare the rates of two identical atomic clocks moving with respect to one another, special relativity predicts that we should observe the transverse Doppler effect; similarly, if we compare the rates of two identical atomic clocks moving along different trajectories in a gravitational field, according to general relativity we should expect to observe the gravitational redshift.

However, it is also clear that these two effects are taken to be an empirical confirmation of the time dilation effects predicted by the theory, only if the assumption is granted that two atoms of the same substance are identical clocks, which, if held side by side in relative rest, always reliably measure the same proper time. The problem emerges when one realizes that such material devices are rather complicated physical systems, which behave in conformity to some dynamical laws. Without an adequate knowledge of such laws, there is no way to tell in advance if a given clock is in fact an ideal clock, whose functioning is not significantly affected by the presence of accelerations, 
electromagnetic fields, or by the gravitational field itself. Thus, it can be objected that what we actually compare with experience is not simply the predicted 'geometrical' effect of time dilation, but the latter together with the physical laws governing the natural process that we have chosen as a clock.

In this sense, Einstein expressed early on the conviction that in relativity theory, as in any physical theory, one seems to be entitled to expect a dynamical explanation of how the measuring instruments work, possibly without calling on other branches of physics (Butterfield, 2002). The initial 'black-boxing' of rods and clocks as unstructured entities was only a provisional compromise, and though probably unavoidable, it was a 'sin' that should not be forgiven. In principle, the dynamics of both rods and clocks, and the other physical systems, should be describable within the framework of relativity theory. As we shall see, however, what requires a dynamical explanation is not the physical process of, say, the slowing down of clocks, but the very existence of identically constructed (gleich beschaffene) clocks that always measure the same true proper time in every circumstance.

In my opinion, in order to properly appreciate this point, which has sometimes been misunderstood in the current philosophical debate, it is important to realize that the entire discussion of the role of rods and clocks in relativity theory turned on a tacit, but fundamental assumption. Max Born once aptly called it (although only in passing and only referring to special relativity), "the principle of the physical identity of the units of measure" (Born, 1920, 191; my emphasis; tr. 1922, 211). The importance of such a principle, in spite of some illuminating works on this issue (Pierseaux, 2003; see also Pierseaux, 1999), has not been sufficiently recognized in the philosophical literature (Brown mentions it, but only in a footnote; Brown, 2005, 81, fn. 41; see also Valentini, 2008, 135-136). However, I would dare to claim that the fate of special and general relativity as physical theories - and not only as the mathematical manipulation of quantitiesdepends entirely on the validity of such a principle, and should thus be considered one of the central issues, if not the central issue of the epistemology of relativistic geometry. In order to make relativity theory physically meaningful, we have to assume the actual existence in nature of physical processes, permitted by some physical laws, that can be used to define and reproduce the units of length and time at different space-time points.

Thus, the epistemological question emerges of whether a theory should be able to describe material objects and processes that serve as its means of verification, a position shared by most relativists, or whether such a description should lie outside the domain theory, a position that, as can easily be guessed, prevailed among physicists working on quantum mechanics (cf. e.g. Peres and Zurek, 1982). Einstein's vacillation between 'phenomenological' and 'dynamical' treatments of rods and clocks should be seen as the attempt to position himself between these two extremes, which can be considered as paradigmatically incarnated by Weyl and Pauli. The real meaning of Einstein's arguments, as often happens in the history of science, can only be understood when one realizes they were formulated in response to certain interlocutors (Beller, 1999). From this point of view, Einstein's unease about the treatment of rods and clocks as unstructured entities is extraneous to the concerns of the supporters of 'Lorentzian pedagogy' in special or general relativity, as well as to those of their opponents. Rather, Einstein's unease should be understood against the background of a truly philosophical question, which - to use his own rhetorical hyperbole-is nothing but "Pilate's famous question: 'What is truth?'” (Einstein, 1949b, 676).

\section{1917-1920. Einstein on Rods and Clocks in Private Correspondence}

Responding to his former student, W. Dällenbach, at the beginning of 1917, Einstein was ready to admit in private correspondence that his treatment of material rods and ideal clocks as unstructured entities was a provisional compromise, which would later be eliminated when a suitable theory of their material structure was found. In the years immediately following, Einstein was forced to further articulate his epistemological standpoint when pressed by the competing objections of Weyl and the young Pauli.

\subsection{Einstein's 1917 Letter to Dällenbach}

In a letter dated 31 October 1916, Einstein made a passing remark to his friend Michele Besso which, in subsequent years, would engender turned out to be a matter of considerable debate: "Your comment about the equivalence of physic[ally] different measuring rods and clocks (and subjected to different prehistories) is fully correct." However, Einstein points out, "this assumption also tacitly plays a role in Galilei-Newton's theory" (Einstein to Besso, 31 October 1916, CPAE//, Vol. 8, Doc. 270,349 , my emphasis). Judging from the tone of this passage, Einstein seemed to consider this hypothesis sufficiently natural (in spite of the fact that, as we shall see, the existence of such 'ahistoric' objects is rather implausible in the classical framework), and not worthy of being addressed explicitly. 
The physical significance of our measurements arises from the fact that, if we measure distances and time intervals with equivalently-constructed instruments, we obtain results that, within a certain experimental imprecision, are equivalent and do not depend of the prehistories of such instruments.

In pre-general-relativistic theories, rods and clocks are used to directly measure coordinate differences (at least in those coordinate systems for which the laws assume their simplest form), assuring their physical significance. A 'body of reference' can in principle be physically constructed as a cubic grid made of rigid rods at relative rest with clocks at the nodes, synchronized using light rays (cf. e.g., Einstein, 1905, 892; 1907, 437). In general relativity, devices measuring space-time are not to be admitted as the physical counterparts of the 'coordinate distances', which are no longer physicallyobservable quantities. Still, it is assumed that the so-called line element $d s^{2}$ has a 'natural' distance-a definite, experimentally-determinable value (up to a constant which can be set $=1$ ), that can be directly measured with 'transportable' rods and clocks that always measure the same velocity of light $\mathrm{c}$ in vacuo (Einstein, 1913, 494, 1913, 494). ${ }^{1}$

Concretely, to measure the magnitude of the $d s^{2}$, we might use, say, a rock salt crystal as a rod and a cadmium atom emitting its red line as a clock. We can arbitrarily choose a certain number of spacings between the atoms of the crystal as a unit of length, or number of wave crests emitted by the radiating atom as a unit of time. It is tacitly assumed that the ratio of such units is an absolute constant, ${ }^{2}$ which is not affected by the gravitational field (i.e., more precisely, it does not depend on the second derivatives of the $\left.g_{\mu v}\right)^{3}$, and possibly by other external influences (in particular the presence of an electromagnetic field).

As we shall see, choosing this kind of physical system is not a casual decision. There was overwhelming spectroscopic evidence, in spite of the missing theoretical explanation (Bohr, $1913 \mathrm{a}, \mathrm{b}, \mathrm{c}$ ), that atoms of the same kind are absolutely identical; the same astonishing identity is shown by the atomic lattices of the crystal of a given substance, as revealed by X-ray diffraction (Laue, Friedrich, and Knipping, 1913; Born, 1915). Such physical systems might therefore adequately play the role of 'identically constructed' rods and clocks, which can be used to reproduce identical units of measure at the remotest places and times, and thus to physically normalize the $d s^{2}$ as the unit interval.

At the same time, the use of atoms or crystalline structures as clocks and rods makes it intuitively clear that these measuring devices are not unstructured physical systems. Precisely for this reason, some time after 15 February 1917, Einstein had to admit to his former Zurich student Walter Dällenbach, that from an epistemological point of view, the idea that the $d s^{2}$ could be found directly through a measurement done by rigid rods was at most a provisional compromise similar to the 'ponderomotive' definition of the electromagnetic field strengths, in which the electric force is defined as something that causes the motion of an electric charge, and in turn, an electric charge is something that exerts electric force:

\begin{abstract}
Dear Dällenbach!
Your remarks are, in my opinion, to a large extent correct. ${ }^{4}$ Strictly speaking, the concept of $d s^{2}$ evaporates in an empty abstraction: $d s^{2}$ cannot be rigorously considered as the result of measurements, not even in the absence of electromagnetic fields. You have rightly indicated the reasons why it is so. Nevertheless, in a didactically reasonable presentation of the theory the $d s^{2}$ must be so considered, as if it were rigorously measurable. The issue here is analogous to that in electrical science, where the definitions for e and $\mathrm{h}$ are given, even though these definitions do not withstand strict criticism. A logically satisfying presentation can be achieved (a posteriori), so that a single, more complex solution is related to the observed facts. A measuring-rod would then be an atomic system of a certain type that does not play any special role in the theory. Thus a four-dimensional continuum can still be maintained and, in upholding the postulate of general covariance, it then has the advantage of circumventing the arbitrariness in the choice of coordinates (Einstein to Dällenbach, after 15 February 1917, CPAE, Vol. 8, Doc. 299, 391; my emphasis).
\end{abstract}

Surprisingly enough, this early passage contains the ambivalent stance on rods and clocks that Einstein would consistently defend until the end of his life. Measuring rods and clocks are made of ordinary matter, that is, they are constructed of particles kept together by non-gravitational forces, which at that time meant electromagnetic interactions. The description of their behavior inevitably implies the comparison of the forces that are internal to rods and clocks with those that are

\footnotetext{
${ }^{1}$ As Einstein points out in a footnote of the latter writing: "We will make the assumption that this is achievable at all locations and at all times" (Einstein, 1913, 490, n. 1). Einstein justifies this assumption by appealing to the equivalence principle: The outcome of any local experiment (in a freely-falling laboratory) is independent of its location in space-time.

${ }^{2}$ I borrow this from Ludwig Flamm (Flamm, 1916, 451), which makes this presupposition of general relativity particularly clear.

${ }^{3}$ For sake of historical accuracy, I have adhered, as far as possible, to the original notation.

${ }^{4}$ Einstein refers to a private conversation.
} 
external. Such considerations ultimately depend on a theory of the electromagnetic interactions involved in material structure. The length of a crystal and the rate of an atomic clock might be affected by a strong variable electromagnetic field. But also, in the absence of electromagnetic effects, Einstein points out, "we obviously do not know whether the [gravitational] field forces [...] or acceleration, do not (in principle) affect the yardsticks and clocks directly" (Einstein to Dällenbach, after 15 February 1917, CPAE, Vol. 8, Doc. 299, 392).

Einstein's initial treatment of rods and clocks, which made it necessary to introduce them as separate entities in the framework of his theory, was thus a logical defect that ought to be eliminated, even though he had just defended this point of view at length in his popular book on relativity, finished at the end of 1916 (Einstein, 1917a). In general, Einstein seems to have been convinced that a satisfactory theory should not simply postulate the existence of the material structures suitable to be used as measuring devices; but rather, it should deduce it. If possible, this should be done without abandoning the field-theoretical framework of general relativity. Then rods and clocks would not play 'any special role in the theory', as opposed to all other physical objects.

However, in Einstein's theory, the role of matter as the source of the gravitational field was an external ingredient not determined by the theory, but rather was something to be prescribed independently, leaving open the question of whether the theories of the electromagnetic field alone, or possibly the electromagnetic field and the gravitational field together, would form a sufficient basis for a theory of matter. ${ }^{5}$ After some initial doubt (Einstein, 1916a), the letter to Dällenbach shows that Einstein began to nurture some hope that general relativity might constitute the starting point for building a field theory of matter; however, he was open (Stachel, 1993) to considering an atomistic alternative, that is, to drop the 'continuum' and along with it, the principle of general covariance. Einstein offered to Dällenbach a balanced analysis, pro and con, of both points of view:

However, you have also understood correctly the disadvantage attached to a continuum. If the molecular interpretation of matter is the correct (practicable) one, that is, if a portion of the world must be represented as a finite number of moving points, then the continuum in modern theory contains way too many possibilities. [...] Indeed, I see fundamental difficulties here [abandoning the continuum] as well. Electrons (as points) would be final conditions (building blocks) in such a system. Do final building blocks exist at all? Why are they all similar in size? Is it adequate to say: God in His wisdom has made them all the same size, each like any of the others, because He so wished? If it had suited Him, He could also have made them dissimilar. Thus we are better placed with the continuum conception, because elementary building blocks do not have to be provided from the outset (Einstein to Dällenbach, after 15 February 1917, CPAE, Vol. 8, Doc. 299, 392; my emphasis).

Thus, even if Einstein left open the possibility of a 'molecular interpretation of matter', where electrons are final 'building blocks', he seemed to be cautiously inclined to search for a continuum theory in which the ultimate constituents of matter, the electrons or ions, would be treated as 'solutions' to some non-linear field equations. ${ }^{6}$ In this context, it would then be possible to find some 'more complex solutions' of such equations that would present characteristics making them suitable to be used as rods and clocks. Rods and clocks would not be introduced 'by hand', but would be treated as a physical system like any other. In a possible general-relativistic theory of matter, the dynamics of both rods and clocks and all other material systems would then be described by the same theory.

As we shall see, this would raise the question of how the theory would be related to reality, that is, in which sense we could claim that the prediction made by the theory, e.g., about the values of the $g_{\mu v}$, could be said to be 'verified' or 'falsified' by observing the behavior of rods and clocks. Although

\footnotetext{
${ }^{5}$ Einstein's theory was consciously formulated regardless of "specific assumptions about the constitution of matter" (Einstein, 1916b, 810), that is, of everything that is not the gravitational field, including the electromagnetic field. In particular, Einstein rejected the "unfounded hypotheses about the structure of the electron or matter" (Einstein to Weyl, 23 November 1916, CPAE, Vol. 8, Doc. 278, 365) that had motivated Hilbert's theory. Hilbert had in fact attempted, along the lines of Gustav Mie's thinking, to use general relativity to construct a theory of matter, explaining "hitherto hidden processes in the interior of atoms" (Hilbert, 1915, 407). One can find here the model of a theory that deduces the properties of atomic systems, that in turn can be used as clocks. Referring to the fact that an atom always measures the proper time $d \tau$. Hilbert pointed out that, "this assumption has only a provisional character"; when physics is finally "fully developed, then the axiom must appear as a consequence of the general theory" (Hilbert, 1916/1917, 284; my emphasis).

${ }^{6}$ Already in January 1908, Einstein expressed his concern about the fact that Maxwell-Lorentz electrodynamics had to postulate the existence of electrons and their laws of motion separately. He argued that, "in my opinion a satisfying theory should so be constructed that the electron appears as a solution" (Einstein to Sommerfeld, 14 January 1908, CPAE, Vol. 5, Doc. 73, 88). Starting in 1909, Einstein attempted to formulate a nonlinear theory of the electromagnetic field, hoping to derive the atomistic properties of both radiation (light quanta) and matter, so that the electron would cease to be "a stranger in Maxwell-Lorentz Electrodynamics" (Einstein, 1909a, 192; see also Einstein, 1909b). In particular, in order to derive the existence of electrons of the same mass and charge, he came to recognize that it was sufficient to include in his equations some constant with dimensions of a length (cf. McCormmach, 1970, for more details).
} 
Einstein had addressed a similar issue previously, at least in passing, ${ }^{7}$ in the following years he was forced to respond to this problem in a more systematic way (Hentschel, 1992a).

\title{
2.2. Rods and Clocks in the First Edition of Weyl's Raum, Zeit, Materie
}

The letter to Dällenbach provides significant textual evidence, the importance of which, as far I can see, has not been sufficiently emphasized in the literature. ${ }^{8}$ Here, Einstein's position on the role of rodand clock-like material systems in relativity theory was already clearly defined in all its essential elements, along with his stance toward the possibility of developing a relativistic theory of matter. In particular, we can already see here that the key to reconciling Einstein's more careful stance with his repeated emphasis on the ideal of 'geometry as a branch of physics' concerning the behavior of physical rods and clocks, was to emphasize the 'provisional' aspect of the latter view (cf. Howard, 2005). As I will try to show, although Einstein was forced by various interlocutors---and by his own pursuit of a field-theoretical approach to the problem of matter---to address this issue mainly in semi-popular writings he would never change his stance towards it.

It could be significant that the letter was written to Dällenbach. Since 1916, he had been working on his doctoral dissertation at the ETH Zurich on a generally covariant extension of Lorentz electrodynamics (see below in section 2.3) under the guidance of the mathematician Hermann Weyl. Returning from the war, Weyl had immersed himself in the study of Einstein's theory (Weyl, 1917; cf. Weyl, 1955, for Weyl's later recollections). Dällenbach's objection to Einstein possibly reflects discussions he may have had with Weyl himself. In fact, during the summer term of 1917, Weyl gave a series of lectures on Einstein's theory, which would be published a year later as Raum, Zeit, Materie (Weyl, 1918a), destined to become one the first and most influential books on relativity. Here, Weyl expressed his reservations about Einstein's treatment of rods and clocks as unstructured entities in special relativity.

Weyl seems to have believed that special relativity could make predictions about the behavior of unaccelerated clocks and rods without a detailed knowledge of the dynamical laws governing their behavior, but only by assuming the Lorentz-invariance of such laws. However, he explicitly pointed out that such 'agnosticism' is no longer allowed when the dynamical systems that we use as rods and clocks undergo acceleration. However, the assumption of the existence of 'memoryless' rods and clocks that are not influenced by their previous accelerations is essential to providing the theory with empirical content.

As Weyl shows, in order to ensure that the global scaling factor of the Lorentz transformations is the unity (cf. Weyl, 1923, 171, for more details), special relativity had to assume that "the static length of a measuring rod" and the "proper time' of the clock" (Weyl, 1918a, 138; tr. 1922a, 176) are the same in all inertial systems. However, there is no other way to determine the equality of lengths or intervals with respect to different inertial frames without accelerating a rigid rod or ideal clock from one frame to another. Thus, an independent assumption is necessary: if one slowed down the rods and clocks again and placed them next to physically identical ones that rest in the new inertial system, there would be no difference in length and ticking rate. ${ }^{9}$ In Weyl's own words:

\begin{abstract}
We require of a rigid body that is to be used for purposes of measurement (in particular, a linear measuring rod) that, after coming to rest in an allowable system of reference, it shall always remain exactly the same as before, that is, that it shall have the same stationary measures (or stationary length); and we require of a clock that goes correctly that it shall always have the same proper-time when it has come to rest (as a whole) in an allowable system of reference. We
\end{abstract}

\footnotetext{
${ }^{7}$ Already in 1910/1011 — in the lecture notes for a course on electricity and magnetism at the University of Zurich—Einstein seems to have argued, referring to the question of the definition of field strength via unit charge, that the idea of a physical theory should not be regarded as "a conceptual system whose individual parts" might "correspond immediately to experiential facts" (CPAE, Vol. 3, Doc. 11, 325[p. 12]; my emphasis), but as a theoretical construction that "is true or false, i.e., corresponding or not corresponding to experience, only as a whole" (CPAE, Vol. 3, Doc. 11, 325[p. 13]; tr. in Howard, 1994, 91-92). As Don Howard showed in a classic paper (Howard, 1990), this attitude might show the influence of Pierre Duhem (cf. also Howard, 2014, 360). See also below, footnote 14. In the following, it will become progressively clearer that the whole discussion on the role of rods and clocks in relativity theory should be understood against the background of this opposition between 'individual parts' vs. a 'whole system', rather than between principle vs. constructive theories.

${ }^{8}$ It is, for instance, never mentioned in the otherwise very careful reconstructions provided by Ryckman (2005) and Fogel (2008). Stachel (1993) analyzes the letter but only considers Einstein's stance toward the continuum/discontinuum alternative.

${ }^{9}$ Einstein explicitly pointed this issue out in a footnote of his 1907 Jahrbuch-paper, after having discussed the problem of the global scale factor in the Lorentz transformations (Einstein, 1907, 419-420): "[ $\mathrm{t}]$ his conclusion is based on the physical assumption that the length of a measuring rod or the rate of a clock do not undergo any permanent changes if these objects are set in motion and then brought to rest again" (Einstein, 1907, 420, fn. 1; my emphasis; see also Einstein, 1910, 156, fn. 2 and Einstein, 1911b, V). Einstein had to assume that the influence of acceleration on rods and clocks "may be neglected" (Einstein, 1907, 455), so that their lengths and ticking rates do not depend on the "prehistory of their motion" (Einstein, 1907, 429, fn. 1). Under this assumption, it is possible to use the same measuring rod and the same clock in different inertial systems (cf. also Lorentz, 1910). Brown famously labeled this assumption the 'boostability' of rods and clocks (Brown, 2005, 30, 81, 121).
} 
may assume that the measuring rods and clocks which we shall use satisfy this condition to a sufficient degree of approximation. [...] The limits of acceleration within which this assumption may be made without appreciable errors arising are certainly very wide. Definite and exact statements about this point can be made only when we have built up a dynamics based on physical and mechanical laws (Weyl, 1918a, 138-139; tr. 1922a, 176; my emphasis).

According to Weyl, the existence of rods and clocks, which are not influenced by the accelerations they may have been subjected to, can be accepted with a reasonable confidence under suitable circumstances. However, strictly speaking this assumption cannot be fully justified without relying on the detailed knowledge of internal dynamics of the physical systems that we use as space-time measuring devices.

The very notion of a rigid body preserving the same length, Weyl explains, is rather problematic in special relativity. ${ }^{10}$ In particular, no body can remain rigid while being continuously accelerated to another 'allowable' system; if we push the body at the same moment at different points, the motion will only gradually be communicated to the whole body, since no signals with arbitrarily high velocities are allowed. Hence, in special relativity, "no body exists which remains objectively always the same no matter what influences it has been subjected to" (Weyl, 1918a, 138; my emphasis; tr. s.m. 1922a, 176); we can speak at most of the 'rigid motion' of a non-rigid body. The rigid motion of an extended body is achieved by careful application of forces to different parts of the body; thus its transfer from one inertial system to another cannot be discussed without entering into dynamical considerations, based on some model of the body and of the effects of acceleration on its shape.

We are in no better position when we consider the behavior of ideal clocks, even if the difficulties due to their spatial extension can be neglected. The claim that a clock records the passage of proper time $\int d s$ along its trajectory is equivalent to what Arnold Sommerfeld has called the "(unprovable) assumption" (in Blumenthal, 1913, 71; cf. Arthur 2010) that one can ignore the effects of acceleration on its internal workings. More precisely, such an assumption depends on our knowledge of the physical constitution of the clock. Mechanical clocks - pendulums or balance wheels-might not be very resistant to strong accelerations (such as smashing them against an obstacle); even an atomic clock accelerated by means of a strong electric field might be ionized and thus stop working as a clock. ${ }^{11}$

According to Weyl, without any detailed knowledge of the internal constitutive arrangement of the material structure of rigid rods and clocks, it is only possible to make a reasonably plausible guess, valid only for quasi-stationary motions, that is, for slow accelerations. In good conscience nothing can be asserted about what happens during arbitrary acceleration. In this sense Weyl speaks of an 'adiabaticity' assumption analogous to slow processes in thermodynamics. When a gas is warmed sufficiently slowly (strictly speaking, infinitely slowly), it will pass through a series of thermodynamic states of equilibrium; similarly, "only when we move the measuring rods and clocks steadily, without jerks, will they preserve their static lengths and proper-times" (Weyl, 1918a, 138; tr. 1922a, 177).

Summarizing, Weyl is making a simple point here upon which, later, most relativists would substantially agree. In special relativity, coordinate differences are said to be measured by means of rigid rods and clocks. However, in doing so we actually use certain concrete physical processes governed by dynamical laws. Only the knowledge of such laws would allow an explanation of the proper behavior of rods and clocks, removing the need to postulate it. In particular, Weyl makes it clear that what has to be explained dynamically is, not only the resistance to acceleration of individual rods and clocks, but the very existence of a class of rods and clocks of identical construction, which remain physically the same despite the influences they were subjected to. This is the fundamental condition of the experimental testability of the theory.

It is in fact precisely because "a sodium-molecule which is at rest in an allowable system remains objectively the same," that we can predict that " $v$ ', the frequency of a sodium-molecule moving with a velocity v", will differ by a Lorentz factor from "the frequency $v$ of a sodium-molecule which is at rest", if "both frequencies are observed in a spectroscope which is at rest" (Weyl, 1918a, 146; my emphasis; tr. s.m. 1922a, 185). Then, if we compare the rates of two identical atomic clocks that are, moving with

\footnotetext{
${ }^{10}$ As is well known, this was shown by Born (1909), whose definition of rigidity (the so-called 'Born rigidity') was later taken up by Paul Ehrenfest (1909), Gustav Herglotz (1910), Frittz Noether (1910) and Max von Laue (1911). Einstein never directly participated in the discussion, which, however, might have played a relevant role in forcing him to abandon the notion of a rigid 'body of reference' (Stachel, 1989; Maltese and Orlando, 1995).

${ }^{11}$ After a talk in Zurich in 1911, Einstein (1911a) confessed that, "according to the theory of relativity we do not know what happens" (Einstein, 1911b, V) to an accelerated clock (on this point cf. Brown, 2009). We can, for instance, imagine it moving along approximate polygonal path composed of $n$ successive small linear paths in which it moves in uniform motion. However, "[t]he sudden change of direction could produce an immediate change in the positions of the hands of the clock [Uhrzeigerstellung]" (Einstein, 1911b, V). Thus, Einstein limited his considerations to very slow accelerations: "the influence of such sudden change should recede the longer the clock [...] moves uniformly, i.e. the bigger the dimensions of the polygon are" (Einstein, 1911b, V). Cf. also Einstein's 1912-1914 review of electrodynamics, CPAE, Vol. 4, Doc. 1, 46[p. 29-30].
} 
respect to one another with the help of light signals, we might observe (or not) a time dilation effect predicted by the theory. However, this comparison is only meaningful under the assumption that, if the two atoms were carefully slowed down and put next to each other they would always tick at the same rate. $^{12^{11}}$

If the existence of physical systems with such characteristics is well supported by empirical evidence, it would be preferable to have some special-relativistic theory of matter that admits certain discrete solutions of a special character, such as static or periodic solutions, that make them suitable to be used as rods and clocks. However, Weyl points out, "the Maxwell-Lorentz theory", the most immediate candidate for a matter theory, being linear, is incapable of solving "the problem of matter" (Weyl, 1918a, 162; tr. 1922a, 203; slightly modified), that is, to account for the existence of electrons, which have a definite invariable mass, and, in addition, a definite invariable charge.

In particular, it is incomprehensible why the separate parts of the negative or positive charge do not repel each other, without introducing some non-electromagnetic force exerting a "cohesive pressure" (Weyl, 1918a, 160, 162; tr. 1922a, 200, 208) of tremendous strength (Poincaré, 1906). Thus the electrons are treated "as something given a priori as a foreign body to the field" (Weyl, 1918a, 165; tr. 1922a, 206). Weyl did not hide his (clearly too optimistic) hope that it was possible to build a "purely dynamical view of matter", à la Gustav Mie (Mie, 1912a, b, 1913), in which matter would turn out to be an "offspring of the field", rather than "its carrier" (Weyl, 1918a, 162; tr. 1922a, 203). Atoms and electrons would not be regarded as "ultimate invariable elements" (Weyl, 1918a, 162; tr. 1922a, 203), but as stable 'knots', particle-like solutions of some set of Lorentz-covariant non-linear partial differential equations (cf. Scholz, 2006, for more details).

Weyl nurtured some hope that such a theory would explain the existence and properties of electrons in electromagnetic terms, and indirectly, those of atoms. In this way one would also be able to account for the stability of atomic spectra that one can use as clocks, or the distribution of atom patterns in a rigid crystalline structure that one can use as rods. However, in the absence of any reliable relativistic theory of matter that would assure the existence of localized stable structures suitable to become space-time measuring devices, Weyl was more inclined to avoid the use of rods and clocks as measuring instruments altogether. "Since the behavior of rods and clocks is to some extent problematic for the establishment of physical laws" (Weyl, 1918a, 139), one may make use of less structured entities and derive the metric structure (up to the choice of a unit of measure) from the observation of light rays and free-falling particles alone (provided certain global assumptions about the entire space-time are added); such measuring devices could be described within the framework of special relativity, or by the available special-relativistic field theories, such as Maxwell electrodynamics (cf. Weyl, 1918a, 139-140).

Thus, in the first edition of his book, Weyl had already sketched the main lines of an epistemological ideal to which he would remain faithful until the end of this life (for an excellent philosophically-oriented overview of Weyl's work, see Bell and Korté, 2014). A theory, according to Weyl, should in principle avoid the use of measuring devices, the behavior of which is extraneous to the framework of the theory (see below, section 5.3). It is, after all, the theory that tells us which physical objects, should they exist, can be used as suitable measuring devices. For instance, our knowledge of the electric field is admittedly only inferred from the behavior of matter, which is the only seat of charge (cf. Weyl, 1918a, 60). In particular, we define the electric field strength in terms of the 'ponderomotive' force on the unit charge. However-Weyl would use this example again and again in his epistemological writings - this definition can be regarded only as a provisional starting point. It is sufficient to consider the fact that we can empirically determine the charge of the test body only by observing the curvature of its path in an electric field, as determined by the Lorentz force law. Thus we have to know the momentum of the charged body at any given time, as well as the strength of the electric field, to calculate its charge.

In general, Weyl insists, it does not make sense to "test a single law detached from this theoretical fabric [Gefüge]" (Weyl, 1918a, 60; tr. 1922a, 67). We need a "whole network of theoretical considerations to arrive at an experimental means of verification" that constitutes a self-supporting system of cross-references: only this "inseparable theoretical whole" (Weyl, 1918a, 60; tr. 1922a, 67) can be meaningfully confronted with experience. Needless to say, Weyl took the same attitude towards the role of the behavior of rods and clocks in general relativity. A proper description of their behavior

\footnotetext{
12 The use of quantum mechanical systems (moving positive ions) as fast moving clocks was suggested by Einstein in 1906 (Einstein, 1906). The reason for this choice (beside the fact that ions in canal rays move very fast) was that we can reasonably assume all ions are identical, so that their spectral lines as measured by a co-moving spectrograph are always the same. Then we can consider such an ion as a clock of definite frequency; "this frequency is given, for example, by the light emitted by identically constituted ions at rest with respect to the observer" (Einstein, 1907, 422; my emphasis). It is worth emphasizing Einstein's claim that ions can be interpreted as 'identically constructed' clocks. Eddington (1918) makes particularly clear that the dimensions of hydrogen atoms at rest are assumed to be the same in all inertial systems. This assures the correspondence of the measuring units in different frames.
} 
would require not only Einstein's gravitational-geometric theory, but a theory of matter as wellwhich, however, general relativity was unable to provide.

So, for instance, one could consider general relativity as empirically adequate, because the predicted redshift of solar atoms is observed. The gravitational redshift again presupposes, however, that two "sodium atoms at rest", those placed in the photosphere of the sun, and the ones on the earth, "are objectively fully alike" (Weyl, 1918a, 197; my emphasis; tr. 1922a, 246). That is, they must emit the same number of wave crests in a given time (if measured by a spectrograph relatively at rest). However, the very existence of identical atoms is not intelligible from the point of view of general relativity, which, like special relativity relies on a merely phenomenological theory of matter "in which the atomic fine structure of matter is disregarded" (Weyl, 1918a, 216).

Due to the impossibility of providing a general-relativistic theory of matter that accounts for the existence of the very structures we use as rods and clocks, Weyl insisted again that it would be more accurate to use testing procedures that involve less dynamical theory. In particular, Weyl initially believed that in general relativity, light signals would suffice to determine the geometry of space-time. Two $g_{i k}$-systems that agree on light rays would determine the same space-time geometry up to a constant $\lambda$ : "the arbitrary remaining proportionality factor can only be determined through the individual choice of a unit of measure" (Weyl, 1918a, 183). After this arbitrary choice has been made once and for all, it is possible to assign specific values to time and space intervals. ${ }^{13}$

\subsection{Weyl's Theory and the Einstein-Weyl Correspondence}

As Weyl would later recall (Weyl, 1946), it was during the 1917 lectures on relativity when he realized that the assumption of the constancy of the scale factor $\lambda$-and thus of the global availability of the units of length and time - was only a contingent feature of the Riemannian geometry adopted by general relativity, an assumption that in principle could be dropped. Following mainly Tullio Levi-Civita (Levi-Civita, 1916), Weyl interpreted the length $d s$ of two adjacent events as an infinitesimal vector in space-time, whose components are the coordinatedifferentials $d x_{i}$ of its end points. In Riemannian geometry, in the general case, a vector transported without changing its components (i.e. parallel-transported) around a loop returns to its original position with the same length but a different direction (non-integrability of direction); Weyl realized that a coherent non-Riemannian geometry might be built in which such an asymmetry between length and direction is avoided (non-integrability of length; for more details cf. e.g., Scholz, 1994; Vizgin, 1994, ch. 3; Ryckman, 2005, sec. 6.4; Bell and Korté, 2014, 4.1.3).

Weyl sent the drafts of his book to Einstein on 1 March 1918, also announcing a 10-page paper, in which he suggested that, by removing such asymmetry between the length and direction of a vector (Afriat, 2009), one could formulate a theory unifying gravitation and the other known nongravitational interaction, electromagnetism (CPAE, Vol. 8, Doc. 472). The non-integrability of direction would account for gravitation, and the non-integrability of length for electromagnetism. In dropping the comparability of lengths at different points, not only the ten components of the metric tensor $g_{i k}$, but also the four quantities $\varphi_{i}$ appear, which happen to behave like the potentials of the electromagnetic field. Moreover, Weyl had clearly hoped to find an explanation for "the existence of the electron and the peculiarities of the hitherto unexplained processes in the atom that could be deduced from the theory" (Weyl, 1918b, 477-478; tr. O'Raifeartaigh, 1997, 34), and with them the existence of bulky material structures.

When Einstein received the draft of the paper on 6 April 1918, he was famously impressed by Weyl's 'stroke of genius'. However, he confessed he was incapable of overcoming what he labeled the 'measuring rods objection' against the theory, which he should have communicated to Weyl in person during a stay in Zurich in late March (cf. CPAE, Vol. 8, Doc. 498, 710). The well-known objection runs roughly as follows. Einstein immediately attributed a concrete physical meaning to the magnitude of a time-like vector ds connecting two adjacent events: it is the time recorded by a clock (some physical system running periodically), carried by a particle that includes those two events in its history.

Put this way, Weyl's theory would predict that the ratio of the rates at which any two physical clocks tick is not constant, but is affected by the electromagnetic potentials $\phi$ they have encountered. A clock would measure its proper time $\int \mathrm{ds}$ in absence of an electromagnetic field but it

\footnotetext{
${ }^{13}$ It must be noted that in a paper finished in October 1917 and published in March 1918 (just about the time the drafts of Weyl's book were finished), Erich Kretschmann had already shown that one must also use free-falling particles to force the factor $\lambda$ to be an arbitrary constant, and not a function of position. Appealing to the constant curvature of the spatial slices of the world in Einstein's new 'cylindrical' cosmological model, one can eliminate this degree of freedom as well, by normalizing the constant factor $=1$ so that curvature of space is constant (Kretschmann, 1918, sec. 23). This solution of the problem, as we shall see, would be brought to the most radical speculative implications by Weyl himself (and Eddington).
} 
would measure $\int \varphi_{v} d s_{2}$ where $\varphi_{v}$ is a function of position) if it has been placed for a long time, say, in a charged metallic box. In this way, however, the rate of the clocks' ticking would depend on the physical circumstances they have encountered in the past. The equality of two line elements, $d s_{1}$ and $d s_{2}$ (in Einstein's notation), would not be 'physically' definable in a univocal way. As a consequence - as Einstein wrote to Weyl on April 1918- "Rel. Theory would fully lose its empirical basis" (Einstein to Weyl, 15 April 1918, CPAE, Vol. 8, Doc. 508, 721).

Since the Prussian Academy only agreed to publish Weyl's paper together with Einstein's objection (cf. CPAE, Vol. 8, Einstein to Weyl, 15 April 1918, Doc. 509), Einstein attached to a letter he wrote Weyl on 19 April (CPAE, Vol. 8, Doc. 512) a detailed response (later published as Einstein, $1918 \mathrm{~b}$, from which citations are taken). Light rays, Einstein claimed, are not sufficient as spacetime measuring devices: "there would indeed be an indeterminate factor left in the line-element $d s$ (as well as in the $g_{i k}$ )" (Einstein, 1918b, 478). This indeterminacy can be removed by using rods and clocks, which allow us to make a global choice of the measuring units. In particular, "a time-like $d s$ can be measured directly by a standard clock whose world-line is contained in $d s$ " (Einstein, 1918b, 478 ), so that $\int d s$ would measure the elapsed time between two arbitrary time-like separated events. This definition would become physically empty, "if the length of a unit measuring-rod (or the rate of ticking of a unit clock) depended on its history" (Einstein, 1918b, 478), as Weyl's theory implies.

Fortunately, nature happens to behave differently. At this point, Einstein presented a powerful argument that would become famous. In particular, he added a fundamental detail that was curiously not emphasized in any previous correspondence with Weyl, explicitly identifying clocks with atomic clocks. From this perspective, if Weyl's theory were true, "chemical elements with spectrallines of definite frequency could not exist" (Einstein, 1918b, 478) and "the relative frequency of two neighboring atoms of the same kind would be different in general" (Einstein, 1918b, 478). In Weyl's theory the final radiuses of, say, two hydrogen atoms separated and then reunited might differ, depending on the different circumstances they might have encountered. As a consequence the spectral lines would not be sharply defined, but should show intermediate values. Experience, however, shows that atoms of a particular element are all 'identical', and can emit only radiation with definite energies, that is, each type of atom gives off a unique set of colors. Frequencies and therefore lengths can be transported in an integrable way. Consequently, "it seems to me that one cannot accept the basic hypothesis of this theory whose depth and boldness every reader must nevertheless admire" (Einstein, 1918b, 478).

Some days later, on 27 April 1918 (CPAE, Vol. 8, Doc. 525), Weyl expressed his intention to write an Erwiderung, a reply, which he sent on 28 April (CPAE, Vol. 8, Doc 526) and would be published alongside Einstein's Nachtrag (Weyl, 1918c). Weyl defended his theory by again expressing the same concerns about the use of rods and clocks as measuring instruments as those found in his book (cf. above section, 2.2).

As he pointed out, even in special relativity it is at all obvious that "a rigid rod always has the same rest-length if it is at rest in an inertial frame" (Weyl, 1918c, 478; my emphasis; tr. s.m. O'Raifeartaigh, 1997, 35) (i.e., the 'world sheet', limited by the world-lines of its end-points is of constant width). No less problematic is the assumption "that under the same circumstances, a standard clock has the same period in standard unit" (Weyl, 1918c, 478-479; my emphasis; tr. O'Raifeartaigh, 1997, 35) (i.e., assuming that $\mathrm{c}=1$ ). For small accelerations, Weyl continues, most real clocks will behave as an ideal clock, that is, they record the passage of elapsed proper $\int \mathrm{d}$ time along their world-lines: however, this "is certainly not the case when the clock (or atom) experiences the effect of a strongly varying electromagnetic field" (Weyl, 1918c, 479; tr. O'Raifeartaigh, 1997, 35). The frequency of an 'electric' clock would be modified by an electric field, displaying, for instance, a 'Stark effect' (the electric analogue of the 'Zeeman effect'), so that their spectral lines would be shifted.

This situation is of course no different in general relativity. Precisely "[b]ecause of this problematic behavior of rods and clocks", Weyl points out, "I have relied in my book Raum, Zeit, Materie only on the observation of light-signals for the measurement of the $g_{i k}$ " (Weyl, 1918c, 479; tr. O'Raifeartaigh, 1997, 35). Weyl erroneously refers the reader to Kretschmann's paper (Kretschmann, 1918) to support his incorrect claim (cf. footnote 12) ${ }^{14}$ But this of course does not affect the epistemological significance of his reservations against the use of entities that are too structured as measuring instruments.

In general relativity, one can at most assume that a clock at rest in a static gravitational field measures "the integral $\int d s$ in the absence of an electromagnetic field" (Weyl, 1918c, 479; tr. O'Raifeartaigh, 1997, 35). But additional assumptions are needed to assure that non-gravitational forces do not affect the internal dynamics of our clocks. In principle, "[h]ow much a clock behaves in

\footnotetext{
${ }^{14}$ In Weyl, 1919b, 194, he specified that free-falling particles are also needed.
} 
arbitrary motion in the common presence of arbitrary gravitational and electromagnetic fields can only be determined by the computation of the dynamic based on physical laws" (Weyl, 1918c, 479; my emphasis; tr. O'Raifeartaigh, 1997, 35). Whether the relative lengths and periods of physical rods and clocks depend on their prehistories or not "has to be justified by an explicit dynamical calculation in both Einstein's theory and mine" (Weyl, 1918c, 479; tr. O’Raifeartaigh, 1997, 36).

For the time being, Weyl could tentatively point out that it was plausible that "an oscillating system of definite structure that remains in a definite static field will behave in a definite way", because "the influence of a possibly turbulent history will quickly dissipate" (Weyl, 1918c, 479; tr. O'Raifeartaigh, 1997, 36), making the effect of the electromagnetic field on the atomic clock behavior practically unnoticeable. Thus his theory was probably not "in contradiction with this experimental situation (which is confirmed by the existence of chemical elements for the atoms)" (Weyl, 1918c, 479; tr. O'Raifeartaigh, 1997, 36).

However, Weyl clearly manifested his conviction that it would be preferable to determine the properties of physical systems that can serve as rods or clocks from the field equations of the theory - which he hoped would be able to account for the granular structure of matter-instead of assuming them separately as primitive entities. Without such a 'dynamical' explanation, "the mathematical ideal of vector-transfer, on which the construction of the geometry is based" has no simple and obvious connection "with the real situation regarding the movement of a clock" (Weyl, 1918c, 479; tr. O'Raifeartaigh, 1997, 36). Weyl considered 'his' geometry to hold in reality because of its conceptual superiority — it is the 'truly infinitesimal geometry' (Weyl, 1918d) - and not because of its correspondence with the behavior of quite complicated material measuring devices (on this topic cf. also Weyl to Einstein, 19 May 18, CPAE, Vol. 8, Doc 544, 767; and Einstein's reply on 31 May 1918, CPAE, Vol. 8, Doc 551, 777).

Again, for our purposes, it is important to emphasize that the feature of rods and clocks that was thought to require a 'dynamical' explanation, was, so to speak, their 'memorylessness', the fact the they stay objectively the same no matter what influences they have been subjected to (cf. Weyl, 1918c, 479). Writing to Einstein on 15 June 1918, Dällenbach, who could not hide his fascination with Weyl's theory, made this point particularly clear. Dällenbach recognized that "there must be some principle", which "connects the different world-points of my experience along a world-line", something "which is presupposed as invariant, as stable", that can be considered "again and again as the same" (Einstein to Dällenbach, 15 June 1918, CPAE, Vol. 8, Doc. 564, 801; my emphasis), allowing the comparison of the length of two tracts along a world-line. However, Dällenbach goes on, "you chose for this purpose such complicated things as 'rigid bodies' or 'clocks'. It seems to me to be arbitrary" (Dällenbach to Einstein, 15 June 1918, CPAE, Vol. 8, Doc. 564, 801).

At the time, Dällenbach was finishing his dissertation under Weyl's supervision (which would be published in October; Dällenbach, 1918). There he tried to develop an electromagnetic model of the stability of matter. Within this framework, matter would be nothing but "a bulk of very many positive or negative electrons" (Dällenbach, 1918, 524), kept together by Lorentz-invariant forces. He believed that this model could be 'easily' generalized to a generally covariant theory. At the time there was no reason to expect that rods and clocks should not be treated as equilibrium solutions of such a theory, just as any other 'bulk' of matter would.

Einstein's answer to Dällenbach (after 15 June 1918) is to my knowledge the best summary of the Weyl-Einstein debate and is worth citing at length:

\footnotetext{
Weyl's theory is wonderful as conception, a genial intellectual achievement [geniale Gedankenleistung], but I'm convinced that nature is different. If two ds at distant points were measured with measuring rods in a certain way, were found to be equal, they would still be found equal, if they were measured in a different way. This is a deep property of our world, which must find expression in the foundation of physics [...] I know that Weyl does not admit it. He would say that clocks and measuring-rods must appear as solutions; they do not occur in the foundation of the theory. But I find: if the ds is measured by a clock (or a measuring-rod), is something independent of prehistory, construction and the material, then this invariant as such must also play a fundamental role in the theory. Yet, if the manner in which nature really behaves would be otherwise, then spectral lines and well-defined chemical elements would not exist (Einstein to Dällenbach, after 15 June 1918, CPAE, Vol. 8, Doc. 565, 803; my emphasis).
}

Thus Einstein admitted again to Dällenbach that in principle it would be better to consider rods and clocks as 'solutions' of some general-relativistic theory of matter, instead of assuming them as unexplained postulates. However, in contrast to Weyl, Einstein believed that for the time being it was sufficient to regard the mathematical invariance of the ds as having a physical counterpart in the surprising 'identity' of atoms that was clearly supported by the spectroscopic data. As he wrote to Besso in August 1918, "otherwise, sodium atoms and electrons of all sizes would exist" (Einstein to Besso, 20 August 1918, CPAE, Vol. 8, Doc. 604, 859; my emphasis). 
At the end of November 1918, Weyl sent Einstein the draft of a new paper on his unified field theory (the revised version was finished in January, and was published as Weyl, 1919a), where he announced the plan to write a revised edition of his book (finished in August and published as Weyl, 1919b), since the first two editions - not least because of Einstein's enthusiastic review (Einstein, 1918a) — had quickly sold out (Einstein to Weyl, 16 November 1918, CPAE, Vol. 8, Doc. 657, 949). After reading the paper, Einstein reiterated his main objection to Weyl's theory (and raised many others). If Einstein could put a finger on the manifest lack of empirical confirmation of the theory, he was, however, also forced to admit that the existence of identical atoms was essential to securing the physical significance of the ds. After all, they were the only physical systems that presented the peculiar kind of identity needed to assure a consistent normalization of the ds: "[ $\mathrm{t}$ ]he existence of spectral lines (of electrons of certain size)", and thus "of clocks independent of their prehistory," "makes it seem natural that from the beginning we can treat the ds as an invariant" (Einstein to Weyl, 21 November 1918, CPAE, Vol. 8, Doc. 661, 954).

It is understandable that for Einstein the issue was of essential importance for the very physical meaningfulness of his theory. As we have seen, it is precisely because all atoms of the same type are absolutely 'identical', on the earth or on the photosphere of the sun, that we can consider redshift observations as an empirical 'test' of general relativity. This effect was, however, very hard to detect (Hentschel, 1994), as the results of the "American measurements" (Einstein to Weyl, 27 September 1918, CPAE, Vol. 8, Doc. 626, 894), performed by Charles Edward St. John, confirmed (St. John, 1917). It is not surprising that by the end of the year on 16 December 1918, scarcely a month after the end of World War I, Arthur Stanley Eddington (who had just finished writing his influential report on relativity; Eddington, 1918), could be tempted to use Weyl's theory to save general relativity from possible refutation. If the redshift measurements failed, Weyl's theory might furnish an explanation, showing that atoms might not consistently measure their proper time if under the influence of an electromagnetic field (Eddington to Weyl, 16 December 1918, Wissenschaftshistorische Sammlungen der ETH-Bibliothek, Weyl Papers, folder 91, p. 522).

\subsection{Einstein, Weyl and the Young Pauli's 'Observability' Criterion}

Einstein and Weyl, despite their dispute concerning the existence of a "central office of standards" (Weyl, 1919a, 103; my emphasis) that would ensure the global availability of units of measure, did agree on the relationship a physical theory should have with the material devices needed for its verification, and possibly about the very program of a field-theoretical approach to the problem of matter. In April 1919, Einstein himself had come to display a more concrete interest in "a theory that will account for the equilibrium of the electricity constituting the electron" (Einstein, 1919a, 349) (something along the lines of Mie's theory), and more generally an interest in "the possibility of a theoretical construction of matter out of gravitational field and electromagnetic field alone" (Einstein, 1919a, 356) (that is, by considering only the quantities $g_{\mu v}$ and $\varphi_{v}$ ).

However, a very different attitude towards these issues was emerging from the German physics scene at about the same time. In April 1919, the 19-year-old student Wolfgang Pauli, who in January had already published a paper on general relativity (Pauli, 1919b), wrote a letter to Weyl, asking him several questions about his unified field theory. Weyl, full of admiration for Pauli's youth, referred him to the forthcoming third edition of his book (Weyl, 1919b), of which he had just received the proofs, and to the second draft of his paper (Weyl, 1919b), to be published in the Annalen der Physik (cf. Weyl to Pauli, 10 May 1919, Pauli, 1979, Doc. 1, 3). Despite Einstein's criticisms, Weyl's program had become even more ambitious. In particular, as he wrote to Pauli, in the paper he had provided a more suitable presentation of the "Electronproblem", even without being able to solve it (cf. Weyl to Pauli, 10 May 1919, Pauli, 1979, Doc. $1,3)$.

On the one hand, he imagined that non-linear field equations - satisfying adequate regularity conditions - should lead to a discrete set of solutions depending on some arbitrary but constant field quantity $\beta$. The problem of matter would then become an 'eigenvalue' problem, where particle-like solutions would be comparable to a set of 'discrete eigenvalues' of a non-linear operator (cf. Weyl, 1919a, 129; cf. Scholz 2006). On the other hand, he expected that Einstein's field equation with the cosmological constant (Einstein, 1917b) should be a consequence of his theory, making it natural to identify this quantity with the radius of curvature of every slice of Einstein's spatially-finite spherical universe. The speculations about the relations between the size of the electron (and thus of the atom) and the size of the universe became Weyl's essential line of defense against Einstein's objection (cf. Weyl, 1919b, 260; for more details see Ryckman, 2005, sec. 6.4.2).

Aside from attempting to answer Pauli's main objection - the fact that the theory was unable to account for the difference between positive and negative charge (cf. Weyl to Pauli, 10 May 1919, Pauli, 1979, Doc. 1, 4) - Weyl encouraged the young student to publish his result independently (cf. Weyl to Pauli, 10 May 1919, Pauli, 1979, Doc. 1, 3). Pauli's paper was finished in July and 
published in October in the Physikalische Zeitschrift. As it appears from a second paper on Weyl's theory that Pauli submitted in November, Pauli initially seems to have been attracted to Weyl's theory precisely because it offered "the possibility to explain and to understand the existence of the electron" (Pauli, 1919c, 461; tr. Mehra and Rechenberg, 1982, 382). As Weyl points out, the conservation laws for charge and mass in classical electrodynamics do not explain why all electrons always have the same mass and charge, including after having interacted with different environments for an arbitrary amount of time (cf. Weyl, 1919a, 128).

However, Pauli added a further epistemological reservation, to which he would return on many occasions in the years that followed (cf. Hendry, 1984):

There is a physical-conceptual objection which should not be forgotten. In Weyl's theory we continuously operate with the field strength in the interior of the electron. For a physicist this [the field strength] is only defined as a force on a test-body, and since there are no smaller testbodies than the electron itself, the concept of the electric field strength in a mathematical space-point seems to be an empty, meaningless fiction. One should stick to introducing only those quantities in physics which are observable in principle. Can it be that we are following a completely false track when using the continuum theories for the field in the interior of the electron? (Pauli, 1919a, 750; tr. Mehra and Rechenberg, 1982, 382).

Pauli introduced here 'observability' as a criterion for the meaningfulness of physical quantities - a criterion that would enjoy great success in some circles (cf. below, section 3.4)-rejecting the continuum theories precisely because they do not satisfy such a criterion.

The young Pauli — who after all was Ernst Mach's godchild — was already displaying here the strong epistemological 'feeling' that the same individual abstract element that occurs in the theory (e.g. the notion of 'field strength') must be directly coordinated with the behavior of physically-existent, even if idealized, objects used as probes (e.g., small bodies of a certain reproducible mass and charge). Unless the quantities introduced into the foundations of the theory can be directly identified with such real objects, the theory is thought to be physically empty. In the Maxwell-Lorentz theory, electrons are needed as the seat of charges, whereas Maxwell's equations are valid only for the 'free field' outside of them. So the notion of an electric field strength inside of the electron is 'an empty, meaningless fiction'. As we shall see, Pauli would need only a few months to explicitly extend this epistemological consideration to general relativity and to any attempt to construct a general-relativistic theory of matter (see below, section 3.2).

The epistemological attitude of the young Pauli elicited a reply from Weyl. In a letter to Pauli, dated 9 December 1919, Weyl defended his program of a unified field theory against Pauli's criticisms, even if he conceded that the problem of matter could probably not be completely solved in a field-theoretical framework without resorting to statistical considerations. In particular, he argued that in some sense it is possible "to measure the fields in the interior of the electron" (Weyl to Pauli, 9 December 1919, Pauli, 1979, Doc. 2, 6), provided that differences inside of the electron "grow to an immediately noticeable magnitude" (Weyl to Pauli, 9 December 1919, Pauli, 1979, Doc. 2, 6), producing some change in its external behavior. He speculated that some change inside of the electron due to its acceleration would account for the fact that electrons in Bohr's stationary orbit do not radiate.

A few days later, on 23 December 1919, Max Born wrote to Pauli that he read "with great interest" his paper on Weyl's theory "in the new issue of the Verhandlungen der Deutschen Physikalischen Gesellschaft" (Born to Pauli, 23 December 1919, Pauli, 1979, Doc. 4, 10). Born confessed to his future assistant that he had been "especially interested" in Pauli's claim that "the application of the continuum theory to the interior of the electron" would be "meaningless, because one is then dealing with things that are unobservable in principle" (Born to Pauli, 23 December 1919, Pauli, 1979, Doc. 4, 10). The way out of "quantum difficulties" (Born to Pauli, 23 December 1919, Pauli, 1979, Doc. 4, 6), Born went on, should be sought while keeping this problem in mind, and without carrying over the macroscopic concepts of space and time into the quantum domain, where length and distance cannot in principle be measured.

It is interesting that Einstein also seems to have taken Pauli's objection very seriously, confessing to Born himself some weeks later: "Pauli's objection is directed not only against Weyl's, but also against everyone else's continuum theory. Even against one which treats the electron as a singularity" (Einstein to Born, 27 January 1920, CPAE, Vol. 9, Doc. 284, 387). However, Einstein, in contrast to Born, did "not believe that one must abandon the continuum in order to solve the [problem of] quanta" (Einstein to Born, 27 January 1920, CPAE, Vol. 9, Doc. 284, 386-387). Even if the space-time continuum could in principle be abandoned, as suggested by Born, Einstein was more inclined to search for "differential equations for which the solutions no longer have any continuum properties" (Einstein to Born, 27 January 1920, CPAE, Vol. 9, Doc. 284, 387). Einstein may have already recognized that in order to pursue a field-theoretical approach to the 
problem of the atomicity of matter, one should drop the epistemological model defended by Pauli and replace it a more sophisticated one. ${ }^{15}$

To Einstein, the failure of Weyl's theory to provide solutions that corresponded to electrons, seemed to be a fundamental failure (cf. Einstein to Ehrenfest, 4 December 1919, CPAE, Vol. 9, Doc. 189, 267-268; and Einstein to Besso, 12 December 1919, CPAE, Vol. 9, Doc. 207, 293). Einstein seemed progressively more convinced that material bodies should turn out to be a mere manifestation of the unique physical reality represented by the field. It is not surprising that Born, in his semi-popular Frankfurt lectures on relativity, which later became a widely-read book (Born, 1920), would adopt the exact opposite attitude: the field is a "mere mathematical device for conveniently describing processes in matter" (Born, 1920, 171; tr. 1922, 190). Before a test body is brought up, the field lines in vacuo have as little physical reality as the lines of latitude and longitude on the sea before, say, someone decides to determine the position of a ship by means of astronomical observations (Born, 1920, 171; tr. 1922, 190).

\section{1920-1923. Einstein on Rods and Clocks in the Public Debate}

Even if Einstein thought that Weyl's theory was flawed, he clearly realized that the independence of rods and clocks from their prehistories was a fundamental presupposition of the empirical meaningfulness of the relativity theories. Einstein was briefly forced to address this issue in public at Bad Nauheim, and more extensively in his famous lecture, "Geometrie und Erfahrung".

\subsection{Rods, Clocks and Born's Principle of The Physical Identity of The Units of Measure}

Even if Einstein was convinced that Weyl's attempt to build a unified field theory was untenable, he had clearly gained an appreciation for the fact that the independence of rods and clocks from their prehistory-which he deemed as sufficiently natural in correspondence with Besso (cf. above, section 2.1) —was not self-evident, yet at the same time was essential to warrant the empirical content of both relativity theories. At the beginning of 1920, in a long, unpublished article intended for Nature on the conceptual development of relativity, Einstein felt compelled to explicitly state that special and general relativity have to assume the "independence of measuring rods and clocks from the prehistory of their motions" (Einstein, 1920a, 280[p. 12]). When "unit measuring rods-compared in relative rest - are found to be equal" (Einstein, 1920a, 280[p. 12]), then they should always be equal when they are compared again. In a note he appended to the manuscript, Einstein insisted that "this sort of equality (also for clocks), one that is enduring and independent of the pre-history of the motion, is a central presupposition of the whole theory" (CPAE, Vol. 7, Doc. 31, note 19).

As we have seen (cf. section 2.1), Einstein was aware of this presupposition no later than the end of 1916 (thus before Weyl's theory was formulated). Weyl's theory had the effect of forcing Einstein to mention explicitly the prehistory condition among the presuppositions of both of his theories. Only if physical systems satisfying this condition really exist in nature can the line element $d s$ be considered a "phys[ically] significant invariant" (CPAE, Vol. 7, Doc. 19, 149[p. 3]).

As Einstein wrote to Cassirer in June 1920, "conceptual systems appear empty to me, if the manner in which they are to be referred to experience is not established" (Einstein to Cassirer, 6 June 1920, CPAE, Vol. 10, Doc. 44, 293). In particular, "[w]ith the interpretation of the $d s$ as a result of measurement, which is obtainable by means of measuring rods and clocks the general theory of relativity as a physical theory stands or falls" (Einstein to Cassirer, 6 June 1920, CPAE, Vol. 10, Doc. $44,293)$. General relativity is a physical theory because it is possible to determine physically the absolute value of $d s$. For example, two time-like ds would be defined as 'equal' if between the beginning and the end of the interval the same number of vibrations emitted by identical atoms occurs. As we have seen, the gravitational redshift, just like the transverse Doppler effect in special relativity, can be taken as an empirical confirmation of general relativity only because different atoms of the same substance can be regarded as identically constructed clocks reproducing the identical unit of time.

Put in more general terms, the empirical verification of the relativity theory presupposes what Max

\footnotetext{
${ }^{15}$ One might speculate that, after all, Einstein had already answered Pauli's epistemological challenge a decade earlier in a series of unpublished lectures on electromagnetism, mentioned above (see footnote 6). Einstein explicitly considered it legitimate to extend the definition of the field strength obtained in vacuo inside ponderable bodies (where the electricity is assumed to be distributed continuously), even if "the force thus defined is no longer immediately accessible to exp[eriment]" (CPAE, Vol. 3, Doc. 11, 325[p. 13]). See also Max Abraham $(1905,22)$ for a similar remark. In other words, "we extend the application of the concept to cases in which the definition [of the field strength in terms of force extorted to small charged bodies] finds no direct application" (CPAE, Vol. 3, Doc. 11, 325[p. 13]). It is in this context that, as I have mentioned, Einstein claimed (just like Weyl, cf. section 2.2), that the individual parts of the theory should not be confronted with experience; only the theory as a whole should be (see above, footnote 6).
} 
Born, in his Frankfurt lectures on relativity mentioned above, labeled the "principle of the physical identity of the units of measure" (Born, 1920, 191; tr. 1922, 211). Even though Born mentioned the principle only in passing (and only in reference to special relativity; cf. Pierseaux, 2003), it does not seem an exaggeration to claim that the validity of the principle is the conditio sine qua non of the physical content of both relativity theories: the quantities that special and general relativity are dealing with posses a physical meaning only if they are consistently measured with the same, reproducible, 'identical' units. Taking distance and duration as basic measurable quantities, as we do in special and general relativity, presupposes the existence of reproducible standards of length and time, of rods and clocks, functioning independently of their prehistories.

Weyl's theory thus had the 'epistemological' merit to suggest that Born's principle could be wrong, or at least that relativity theory gave no reason to assume that it actually holds in nature. This was Besso's point of view, for instance, as can be inferred from Einstein's response to him in a letter dated 20 August 1920:

\begin{abstract}
The remark about Weyl's book refers to his theory of electricity. I understand your view. You think: The invariance [Unveränderlichkeit] of the relative extension of bodies does not need to be put in the foundation of the theory; it would be more beautiful if it comes out as a consequence or acceptable if it had a special place in the theory hypothesis. But do not forget that the [Weyl's] theory is based on a measuring rods geometry. Then it must be assumed that the relative lengths of measuring rods is a function of their prehistory, whereas the real measuring rods and clocks are relatively invariant. Then the measuring rods that one uses in the foundation of the theory are only thought measuring rods [nur gedachte Massstäbe] that behave differently from the real ones. This is repugnant (Einstein to Besso, before 26 August 1920, CPAE, Vol. 10, Doc. 85, 347; first emphasis mine).
\end{abstract}

Einstein was ready to admit that in principle it would be better if 'the invariance of the relative extension of bodies' would come out as a 'consequence' of the theory, as Weyl had suggested. ${ }^{16}$ However, he also saw the possibly unpleasant implications of this view. It would have allowed Weyl to claim that a nonRiemannian geometrical law of vector-transfer - the behavior of the 'thought' rods - obtains at the basis of his own theory, even though the physical behavior of the 'real' rods turns out to be Riemannian. Einstein found this position repugnant precisely because it would imply that the behavior of the 'real' rods contradicts that of the 'ideal' ones'.

\title{
3.2. The Bad Nauheim Debate
}

A month later, at the 86th Assembly of the Association of German Scientists and Physicians (Versammlung der Gesellschaft Deutscher Naturforscher und Ärzte) in Bad Nauheim in September 1920 (famous mainly for Einstein's confrontation with his anti-relativity critics), Weyl pursued for the first time precisely this strategy, which he had anticipated in his 1919 writings (cf. above, section 2.4). He outlined a now well-known speculative explanation for the discrepancy between the behavior of 'ideal' and 'real' rods. Roughly, Weyl suggested that the atoms we use as clocks might not preserve their size if transported, but adjust it every time to some constant field quantity, which he could identify with the constant radius of the spherical curvature of every three-dimensional slice of the world, furnishing a natural unit of length. The geometry read off from the behavior of material bodies would appear different from the actual geometry of space-time, because of the 'distortion' due to the mechanism of the adjustment.

In the discussions at the Bad Nauheim meeting, Einstein maintained his characteristically ambiguous attitude towards the role of rods and clocks in relativity theory. After Weyl's talk, he insisted once again that the "arrangement of [his] conceptual system," "it has become decisive [massgebend] to bring elementary experiences into the language of signs [Zeichensprache]" (Einstein in the discussion of Weyl, 1920, 650). For Einstein, "temporal-spatial intervals are physically defined with the help of measuring rods and clocks", under the assumption that "their equality is empirically independent of their prehistory" (Einstein in the discussion of Weyl, 1920, 650). Einstein insisted that precisely upon this assumption rests "the possibility of coordinating [zuzuordnen] a number ds to two neighboring world points"; if this was impossible, general relativity would be robbed of "its most solid empirical support and possibilities of confirmation" (Einstein in the discussion of Weyl, 1920, 650).

During the discussion following Max von Laue's paper on the redshift, however, Einstein showed a quite different attitude. Laue showed that the coordinate interval $\mathrm{d} \vartheta$ measured by an atom on the sun

\footnotetext{
${ }^{16}$ It is worth noticing that this concern was shared by other relativists at about the same time. Eddington also insisted that "in a strict analytical development the introduction of scales and clocks before the introduction of matter is - to say the least of it - an inconvenient proceeding" (Eddington, 1920a, 152). It would be preferable to "define matter in terms of the elementary concepts of the theory; then we can introduce any kind of scientific apparatus, and finally determine what property of the world that apparatus will measure" (Eddington, 1920b, 191).
} 
is transmitted unchanged by light signals (at least in a static gravitational field ${ }^{17}$ ), so that the redshift emerges by confronting the frequency of such signals with those of an atom of the same type at rest measuring the proper time $d \tau$.

Replying to some concerns expressed by the mathematician Georg Hamel during the discussion, Einstein pointed out that "[s]ince the emitting atoms are to be regarded as a clock in the sense of the theory" (Einstein in the discussion of Laue, 1920), that is, as identically constructed clocks, "then the redshift is one of the safest results of the theory". One can 'verify' that the relation $\mathrm{d} \tau^{2}=$ $\mathrm{g}_{44} \mathrm{~d} \vartheta^{2}$ established by general relativity actually holds in reality. After all, during the same session of the conference, a young Bonn spectroscopist named Leonhard Grebe had communicated very favorable results (obtained in collaboration with Albert Bachem), measuring the shift of the cyanide band of the sun spectrum (Grebe, 1920; cf. Hentschel, 1992b, for more details).

Einstein, however, in the very same sentence, did not hesitate to admit that "[it] is a logical shortcoming of the theory of relativity in its present form to be forced to introduce measuring rods and clocks separately instead of being able to construct them as solutions to differential equations" (Einstein's reply in Laue, 1920, 662; my emphasis). Thus Einstein apparently believed that it would be logically or epistemologically preferable if the field equations of the theory had suitable solutions corresponding to particles, from which in principle the stability of a more complicated, bulky configuration of matter could be reconstructed, including rod- and clock-like structures. In particular, only a few months before, in his famous Leiden talk on aether and relativity (Einstein, 1920b), Einstein had again expressed his preference for a theory in which particle-like matter is nothing but particular "condensations [Verdichtungen] of the electromagnetic field" (Einstein, 1920b, 14), possibly held together by gravitational forces.

Against this field-theoretical approach the young Pauli, in his remarks at Bad Nauheim, reiterated his objection based on his 'observability' criterion. Just as the field strength in the interior of the electron is meaningless because there is no smaller test particle than the electron, "one could claim something similar concerning spatial measurement, since there are no infinitely small measuring-rods" (Pauli in the discussion of Weyl, 1920, 650). The time or space intervals have a physical basis only if there is some actual or possible physical process that has a length or a duration shorter or equal to the space or time interval in question. A distance smaller than the electron would be physically meaningless since there is no physical process that could realize such an interval. The attempt to define the electromagnetic field or gravitational field in the interior of elementary particle to account for their stability should be rejected on epistemological grounds.

Einstein's reaction was apparently evasive. On closer inspection, however, his answer shows that he had become aware of the fact, that, in order to pursue a field-theoretical approach to the problem of matter, the definition of the gravitational potentials in terms of rods and clocks, which he had used against Weyl, was too limited. Einstein pointed out to Pauli that "with the increasing refinement of the system of scientific concepts, the manner and procedure of associating the concepts with experiences becomes increasingly more complicated" (Einstein in the discussion of Weyl, 1920, 650). In particular, he recognized that in cases such as that of the continuum theories, "one finds that a definite experience cannot be associated any longer with a concept" (Einstein in the discussion of Weyl, 1920, 650). According to Einstein, there is an alternative: one can abandon 'continuum theories' for the sake of Pauli's observability criterion, or replace such a "system of associating concepts [with experiences] with a more complicated one" (Einstein in the discussion of Weyl, 1920, 650 ). A decision as to which alternative is more suitable, Einstein pointed out, can only be given on the basis of pragmatic reasons (Einstein in the discussion of Weyl, 1920, 650). However, it is clear that Einstein would opt for the second choice. One should probably view the epistemological considerations that he made in the following years as an attempt to publicly justify this choice.

Pauli's test-particle-objection, together with many others, appeared in a draft manuscript of his contribution on relativity in the Enzyklopadie der Wissenschaften which must have already circulated at Bad Nauheim. Perhaps it was after reading such a draft (cf. Scholz, 2006) that Weyl decided to give up on a possible field theory of matter in the form of Mie's theory (see Weyl's letter to F. Klein on 28 December 1920), and started to conceive of particles as singularities (cit. in Scholz, 2006). However, Weyl did not agree with Pauli on the necessity of assuming rods and clocks as empirical indicators external to general relativity. The 4th edition of Raum, Zeit, Materie (Weyl, 1921a) - finished in November 1920 - introduced the new strategy explained in Bad Nauheim. The idea that real clocks behave according to the laws of Weyl's world geometry is contradicted by the behavior of 'atomic clocks', which emit spectral lines of a definite frequency. Weyl therefore needed to explain "the discrepancy between the idea of congruent transfer and the behavior of measuring-rods and clocks and atoms" (Weyl, 1921a, 280; tr. 1922a, 308).

The mechanism of adjustment is the only way to explain the surprising fact that electrons and

${ }^{17}$ In the general case, the number of vibrations of an atom transmitted by light signals is coordinate-dependent. 
hydrogen nuclei always have the same mass and charge, and thus the very existence of identical atoms, along with the possibly that these atoms, under given external conditions, settle into identical crystalline structures. The fact that all electrons always have the same charge is incomprehensible from the point of view of the Lorentz-Maxwell theory, and had to be introduced as a separate hypothesis. Classical electrodynamics demands the overall conservation of the charge $e(d e / d t=0)$, but the charge of an individual macroscopic body will not generally remain constant given the body's interaction with its environment (cf. Weyl, 1921a, 281).

Weyl thus had good reasons to claim that the only explanation for the fact that electrons always have the same charge, whatever their prehistory, might have been to assume that there is some field quantity of the dimension of a length (i.e., it is simply a number) to which the charge of the electrons 'adjusts' itself in a certain proportion. This was, after all, the general framework that, e.g., Mie (who also gave a talk at Bad Nauheim; Mie, 1920) had tried to realize without any success (cf. also footnote 5). A certain state of equilibrium of the negative (or positive) electricity would always be 'reestablished' whatever disturbance it may have experienced in the past, just as the magnetic needle always points north, despite what may have happened to it previously.

Weyl's distinction between 'adjusting' and 'persisting' quantities moreso expressed a problem than offered a solution. Thus, Weyl never abandoned this distinction, even when he became detached from the very idea of a unified field theory (see below, section 5.3; for a very effective presentation of this distinction see Fogel, 2008, sec. 3.2). The very existence of 'identically constructed' rods and clocks should be "decided on the basis of the actually valid natural laws" (Weyl, 1921a, 281), and not assumed from the outset as a separate hypothesis. After all, Einstein's assumption that physical clocks exist that are fully 'ahistorical' was anything but obvious. Two identical 'classic' atomic systems with different prehistories would probably differ in some small detail due to their interaction with the environment, and their spectral lines would be slightly shifted, so that classically, a spiraling charge should emit light of all colors. Emerging quantum theory had already made clear that the spectral identity of atoms revealed by experience cannot be explained in this framework. The fact that all atoms of the same type are exactly identical clearly cannot depend on an initial agreement established in the past, which has been 'preserved' since then, even though the atoms had encountered very different physical circumstances. It was more plausible to argue that each time they 'adjust' anew to a certain equilibrium value.

According to Weyl, without such a dynamical explanation of their persistence, the use of rods and clocks as empirical indicators should possibly be avoided. On 28 January 1921, he presented to the Gesellschaft der Wissenschaften zu Göttingen a paper in which he proved rigorously-in terms of the relations between conformal and projective structures of space-time- that the metric $g_{i k}$ (and thus the space-time geometry) can be determined (up to a choice of a measuring unit) by the behavior of point particles and light rays. "Measuring rods and clocks are not necessary for this" (Weyl, 1921b, 101). However, two $g_{i k}$-systems agreeing on free-falling particles and the trajectories of light rays might differ by a constant factor (one might still be the scaled replica of the other). Thus, a global definition of the measuring units, implied in the fully Riemannian structure of space-time, was not possible with this method (cf. Ehlers, 1988).

\subsection{Einstein's 1921 Lecture on the Foundation of Geometry}

On 27 January 1921, at the Berlin Academy's Leibniz Day public celebration, Einstein gave an address entitled, "Geometrie und Erfahrung," published in March as a paper and later as a booklet in expanded form (Einstein, 1921a). The influence that Einstein's talk exerted on the history of philosophy of science does not need to be emphasized (cf. e.g. Howard, 2014). It is not at all surprising that Einstein used the lecture as an opportunity to place himself along the epistemological spectrum - whose extreme positions may be best represented by Pauli and Weyl—by using a language that was accessible to the non-specialist, though still 'cultured' public that attended the lecture.

Einstein famously attached "special importance to the view of geometry" (Einstein, 1921a, 126; tr., 1954a, 235) as the study of the laws of the possible disposition of practically-rigid bodies, because "without it I should have been unable to formulate the theory of relativity" (Einstein, 1921a, 126; tr., 1954a, 235). However, Einstein emphasizes that there are good reasons to "reject the relation between the body of axiomatic Euclidean geometry and the practically-rigid body of reality" (Einstein, 1921a, 126; tr., 1954a, 235). The geometrical behavior of physical bodies depends upon temperature, external forces, etc. Thus, as Poincaré showed, if the behavior of bodies contradicts Euclidean geometry, the latter can always be saved by blaming the apparent non-Euclidean behavior of rods and clocks on some distorting influences.

Of course, the supporter of practical geometry could claim that it is always possible to isolate bodies from such distorting influences, and thus "to determine the physical state of a measuring-body 
so accurately that its behavior relative to other measuring-bodies shall be sufficiently free from ambiguity, allowing it to be substituted for the 'rigid' body" (Einstein, 1921a, 127; tr., 1954a, 237). This would imply, however, that one possesses a detailed knowledge of the dynamical laws that describe the material constitution of the measuring bodies. In this sense, Poincaré could rightly point out that "[g]eometry $(G)$ predicates nothing about the behavior of real things, but only geometry together with the totality $(P)$ of physical laws can do so" (Einstein, 1921a, 126-127; tr., 1954a, 236). Using symbols, Einstein famously claimed that "only the sum of $(G)+(P)$ is subject to experimental verification" (Einstein, 1921a, 127; tr., 1954a, 236).

This, of course, is the exact opposite of the strict opposition between axiomatic and practical geometry from which the lecture started. Einstein thus maintains a characteristically ambivalent stance, which is well expressed by this passage:

Sub specie aeterni Poincaré, in my opinion, is right. It is also clear that the solid body and the clock do not in the conceptual edifice of physics play the part of irreducible elements, but that of composite structures, which must not play any independent part in theoretical physics. But it is my conviction that in the present stage of development of theoretical physics these concepts must still be employed as independent concepts; for we are still far from possessing such certain knowledge of the theoretical principles of atomic structure as to be able to construct solid bodies and clocks theoretically from elementary concepts (Einstein, 1921a, 127, my emphasis; tr., 1954a, 236-237).

In spite of the reference to Poincaré, the alternatives that Einstein describes in this passage strongly resemble those he had addressed in the letter to Dällenbach some years before, and later discussed in his correspondence with Weyl.

Sub specie temporis, in the present state of the development of physics, Einstein had adopted the view that rods and clocks should be used to give physical content to the physical quantities that special and general relativity deal with, even though neither theory has the conceptual means to explain the stability of such material systems. Sub specie aeterni, however, rods and clocks should emerge as solutions of a future theory of matter, possibly a field theory encompassing gravitation and electromagnetism that can account for the existence of rod- and clock-like configurations of matter. E.g., a 'rod' is made up of a definite number of atoms held together by electromagnetic forces governed by quite definite physical laws. It could be said to be rigid, that is, to preserve the 'same' length, when, according to these laws, it is expected to 'settle' every time into the 'same' equilibrium configuration. Thus, what is actually compared to reality is only geometry together with these physical laws (cf. Cunningham, 1921, 131, finished in 1920, for a similar remark).

Again, Einstein was clearly pleading for a 'dynamical explanation' of the behavior of rods and clocks; but it is important to emphasize the kind of behavior initially assumed as obvious, and that begged for a dynamical explanation. According to Einstein, "[a]ll practical geometry is based upon a principle which is accessible to experience". Let us call two (irreversible) marks upon a practicallyrigid body a "tract" (Einstein, 1921a, 127; tr., 1954a, 237; cf. Hemmo and Hagar, 2013). Then two tracts are said to be 'equal to one another', that is, have the same length, if the 'marks' of one tract can be brought to coincide with those of the other. An analogous process can be imagined for clocks leaving (irreversible) marks on a dial, which can in some sense be brought to coincidence with marks left by another clock. It must be presupposed that this procedure leads to consistent results:

\begin{abstract}
If two tracts are found to be equal once and anywhere, they are equal always and everywhere [...] The above assumption for tracts must also hold good for intervals of clock-time in the theory of relativity [...]: if two ideal clocks are going at the same rate at any time and at any place [...], they will always go at the same rate, no matter where and when they are again compared with each other at one place. If this law were not valid for natural clocks, the proper frequencies for the separate atoms of the same chemical element would not be in such exact agreement as experience demonstrates. The existence of sharp spectral lines is a convincing experimental proof of the above-mentioned principle of practical geometry. This, in the last analysis, is the reason that enables us to speak meaningfully of a Riemannian metric of the four-dimensional space-time continuum (Einstein, 1921a, 127-128, my emphasis; tr., 1954a, 237).
\end{abstract}

There is little doubt that Einstein is reiterating here his argument in favor of the fact that atoms are reliable clocks, the same argument that he had used against Weyl's theory: the existence of welldefined spectral lines for atoms of the same time, independently of what might have happened to them in the past, provides compelling 'empirical evidence' that the geometry of space-time is Riemannian. ${ }^{18}$ Single atoms of the same element certainly have different histories, but their mass and

\footnotetext{
${ }^{18}$ Michael Friedman, in one of the most cited and influential papers on "Geometrie und Erfahrung" claimed that Einstein's paper should be understood "against the background of a preceding conception of geometry-one that was dominant in the nineteenth century" (Friedman, 2002, 196; see also Friedman, 2010, 2014). More recently Tom Ryckman (2005) has
} 
proper frequencies are always the same (cf. also Einstein, 1921b, 262; Einstein, 1922b, 41).

If the choice of the units of measure is arbitrary (which atom we chose do define the time unit is arbitrary), the ratio of such units can be regarded as absolute dimensionless constants. Under this presupposition we can normalize time and length intervals once and for all, so that the length of the interval between any pair of space-time points can be compared with that of any other in terms of these units. After all, the entire empirical content of relativity is that it makes predictions about the value of length and time differences. For these predictions to be testable empirically, it is essential that the units of measure should be physically reproducible.

Once such an assumption has been granted, then the question of whether the geometry of space is Euclidean or non-Euclidean "must be answered by experience, and [is] not a question of a convention to be chosen on grounds of mere expediency" (Einstein, 1921a, 127; tr., 1954a, 238). Let's consider a small rigid rod, which has the same length in every location and orientation. If its length, over a finite region of space, is determined solely by the coordinate differences of its end points, then the geometry is Euclidean. If this turned out to be impossible, for any choice of coordinates, then the geometry of space would be non-Euclidean, and coordinate distances should be multiplied by the 'conversion factors' $g_{\mu v}$ to be translated into proper distances. In this sense, practical geometry simply summarizes the laws of the disposition of practically-rigid bodies, and can be empirically confirmed or falsified.

In a remark made at Bad Nauheim that without doubt addresses Pauli's point, Einstein admits that such practical approach to geometry would probably fail at the submolecular level. The validity of the practical geometry 'inside' of elementary particles would be based on extrapolation. For instance, we could legitimately raise the question, whether "gravitational fields play a part in the constitution of matter", and thus whether the "continuum within the atomic nucleus [is] to be regarded as appreciably non-Euclidean" (Einstein, 1921c, 409; cf. also CPAE, Vol. 7, Doc. 50, 377), even if in principle there are no rods or clocks smaller than the elementary particles:

It is true that this proposed physical interpretation of geometry breaks down when applied immediately to spaces of submolecular order of magnitude. But nevertheless, even in questions as to the constitution of elementary particles, it retains part of its significance. For even when it is a question of describing the electrical elementary particles constituting matter, the attempt may still be made to ascribe physical meaning to those field concepts which have been physically defined for the purpose of describing the geometrical behavior of bodies which are large as compared with the molecule. Success alone can decide as to the justification of such an attempt (Einstein, 1921a, 127128, my emphasis; tr., 1954a, 237).

Einstein recognizes that this extrapolation might fail, just like the concept of temperature (defined as the average translational kinetic energy of the molecules), cannot be attributed to the molecules themselves. However, he seems to implicitly recognize that in other cases it was possible to extend the use of a concept beyond the domain in which it was initially defined. Clearly, Einstein hoped that the field theory of matter unifying electromagnetism and gravitation might be such a case. For instance, the geometry inside of elementary particles might be considered legitimately as nonEuclidean - even if this claim cannot be 'tested' by using rods and clocks — if this assumption led to a theory that has such particles as stable solutions.

There is, then, enough textual evidence to show that Einstein's lecture can be properly understood only against a hidden dialogical background; the lecture was an attempt to offer an articulate answer to the competing objections raised by Weyl and Pauli.

Against Weyl's theory Einstein pointed out that we have very good reasons to regard atoms of the same substance as identically constructed clocks. As Einstein put it in his Princeton lectures held in May 1921, "this assumption is certainly warranted by experience" (Einstein, 1922b, 41; my emphasis; tr., 1923a, 67). One can verify the predictions of general relativity, e.g., the value of the 'geometrical' quantity $g_{44}$ of the sun's gravitational field, precisely because, if two identical clocks (on the sun and on the earth) measure equal values of $d s$, the values of $d x_{4}$ they measure would differ by a factor $\sqrt{g_{44}}$ (a difference that is responsible for the gravitational redshift).

insisted that Einstein's "Geometrie und Erfahrung" should be read in the context of the Einstein-Weyl debate. I tend to agree with Ryckman, that Einstein's homage to the classical Helmholtz-Poincaré debate should be considered more as an analogy (see also below, section 4). Einstein seemed to be more concerned by the very assumption that the relative

lengths of rods is independent of prehistory, an assumption that characterized all Riemannian geometries, than he was with the problem of making a choice between Euclidean and non-Euclidean types of Riemannian geometries. However, I disagree with Ryckman that the appearance of Weyl's theory was responsible for making Einstein change his mind about the use of rods and clocks as unstructured entities. As I have tried to show, the correspondence with Dällenbach indicates that Einstein's position was fully defined before Weyl's theory was conceived (cf. above, section 2.1). Moreover, as Howard has shown, Einstein was committed to the ideal that only the theory 'as a whole' can be compared with experience, at least in 1910-11 (cf. above, footnote 6 and footnote 14). 
Against Pauli's observability requirement, Einstein was ready to admit that stricto sensu such 'definition' of the $g_{44}$ is not an epistemological necessity, but only an initial compromise. In principle, one can always account for the fact that the redshift is not observed by assuming that the emitting atomic systems on the sun are not 'identical' to the ones on earth, in order to compensate for the appearance of the factor $\sqrt{g_{44}}$ (cf. Silberstein, 1922, 105). One has to keep in mind that what we actually compare to experience is always geometry (the value of the $g_{44}$ ) plus physics (the theory of atomic spectra).

\subsection{The Consolidation of Pauli's Observability Criterion of Meaning}

It is precisely because of these epistemological conundrums that Weyl believed he still had some cards to play. In 1921, the 'pivotal year' for unified field theories (Vizgin, 1994, ch. 4), Weyl (followed to some extent by Eddington, 1921a, b) presented his strategy of 'doubling the geometry' (the real 'aether geometry' and the 'body geometry' distorted by the mechanism of adjustment), in three papers intended for different audiences, finished in February (Weyl, 1921b), May (Weyl, 1921c) and July (Weyl, 1921d). As he put it in his account of the Bad Nauheim debate-which was finished in August 1921 (but published the following year as Weyl, 1922b) - the problem of how "the comparison of the units of the ds in different world locations is possible" (Weyl, 1922b, 52; my emphasis) could not simply be solved by returning to the happy coincidence that identical atoms exist that consistently show the same spectral signature.

However, Weyl had to face a much fiercer opponent emerging as the rising star of German physics. In September 1921, Pauli's encyclopedia article on relativity theory (which was finished in December, but underwent some improvements in April and May) was finally published, as part of the fifth volume of the Enzyklopädie der Mathematischen Wissenschaften, and later as a book with an introduction by Pauli's mentor, Arnold Sommerfeld (Pauli, 1921). The article was unanimously considered a masterpiece, in particular by Einstein himself (Einstein, 1922a). In chapter 5, Pauli reiterated his technical and epistemological concerns about attempts to derive the atomic nature of electricity from the framework of the continuum theories.

Moreover, paragraph 65 amounts to a devastating epistemological 'deconstruction' of Weyl's theory in particular, a critique that was hard to dismiss. If Weyl's theory seeks to make predictions that are closely linked with the behavior of measuring rods and clocks, just like Einstein's theory, then the theory is clearly wrong. Not only should the effect of the electromagnetic field be noticeable in the spectral lines of a given substance, but, as Pauli shows, "the differences would increase indefinitely in the course of time" (Pauli, 1921, 763; tr., 1958, 196). If one renounces this interpretation, as Weyl later suggests (by blaming the Riemannian behavior of clocks on the mechanism of adjustment), then the theory loses its physical meaning, becoming just a mathematical scheme that furnishes only "formal, and not physical, evidence for a connection between [the] world metric and electricity“ (Pauli, 1921, 763; tr., 1958, 196).

Thus, Pauli came to appreciate the definition of Weyl's 'world geometry' - as opposed to a 'natural geometry' by rods and clocks - as a 'graphical representation' of reality, that Eddington had suggested in his celebrated textbook on relativity, The Mathematical Theory of Relativity (Eddington, 1923, dated August 1922), as somehow illuminating (cf. also Eddington, 1922). ${ }^{19}$ As Pauli confessed to Eddington in a letter from September 1923, he found no logical contradiction in Weyl's (or Eddington's) approach, but rather an epistemological shortcoming.

The letter to Eddington probably contains the most extensive expression of Pauli's philosophical credo. In particular, he again compared the definition of the electric field as force on electrically charged test particles with that of the gravitational field in terms of the behavior of rods and clocks:

The notion of field only makes sense if we are able to indicate a reaction, which, in principle, would allow to measure the field strengths at every space-time point, if we so desire. [...] As soon as the reaction ceases to be specifiable or in principle executable, the respective field concept is no longer defined. [...] I adhere to the (of course, unprovable) viewpoint that each physical theory [...] must start with a definition of the field quantities used; the definition should state how these quantities can be measured. [...] (The most beautiful success of relativity theory was indeed that it yielded a deep and tight connection between the results of measurements of rods and clocks, the orbits of freely-falling mass points and those of light rays.) These postulates cannot be proved logically or by means of the theory of cognition [erkenntnistheoretisch]. However, I am convinced that they are correct (Pauli to Eddington, 23 September 1923, Pauli, 1979, Doc. 45, 117-118).

\footnotetext{
${ }^{19}$ For a reconstruction of Eddington's position on this issue and how it differs subtly from Weyl's, see Ryckman, (2005, sec. 8.2).
} 
It must be emphasized that Pauli was not at all in a minority position. Pauli's epistemological attitude turned out to be extremely influential, especially among the Göttingen physicists who started to develop quantum mechanics in the ten years that followed.

It is by appealing to this epistemology that Pauli confessed to Bohr at the end of 1924 that one should consider "observable properties of the stationary states", such as the intensity of spectral lines, as "something much more real than the "orbits"" (Pauli to Bohr, 31 December 1924, in Pauli, 1979, Doc. 79, 197) of electrons around the nucleus in the Bohr-Sommerfeld atomic model. This attitude clearly prevailed among the Göttingen community (cf. e.g., Born, 1925, 114, Born and Jordan, 1925, $479 \mathrm{fn}$.). It is easy to see the very same methodological stance reemerging in the opening sentences of Werner Heisenberg's Umdeutung paper (Heisenberg, 1925; cf. Camilleri 2009, ch. 2).

\section{1923-1930s. The Assessment of Einstein's Two-Stage Strategy}

Against the background of his first contributions to a unified field theory, Einstein, in his semi-popular writings (in particular the Nobel Prize lecture), once again tried to justify his provisional use of rods and clocks to give the geometrical statements of the theory empirical content, even though he agreed that they are complicated physical systems described by rather complicated physical laws.

In his letter, Pauli of course did not hide from Eddington that he had similar concerns about "Einstein's work in the Berliner Bericht", that he had just read (Pauli to Eddington, 23 September 1923, Pauli, 1979, Doc. 45, 115), In early 1923 Einstein published in the Proceedings of the Prussian Academy (Einstein, 1923b, c), to which he later added a paper in English in the September issue of Nature (Einstein, 1923e). By January 1923, on his return trip from Japan, Einstein's had by his own admission become infatuated with Eddington's generalization of Weyl's theory. He wrote to Bohr from the ship: "Eddington has come closer to the truth than Weyl" (Einstein to Bohr, 10 January 1923, CPAE, Vol. 13, Doc. 421, 692). Weyl wrote a letter to Einstein, criticizing Einstein's paper. However, he was pleased to meet Einstein "on the same purely speculative paths" against which he "always protested before" (Weyl to Einstein, 18 May 1923, cit. and tr. in Stachel, 1986b, 240).

In this context, Einstein probably found it even more pressing to give a justification of his attitude toward the relationship between theory and experience. After all, Einstein had on many occasions defended a view à la Pauli, according to which relativity theory has empirical content because its predictions may or may not correspond to the behavior of rods and clocks that actually exist. However, he often pointed out that it was actually "a logical tension [Inkonsequenz]", because one presupposes for the measurements "such complicated structures [Gebilde] as measuring rods and clocks as given, instead of constructing them from theoretical concepts" (Winternitz, 1923, 224). ${ }^{20}$

During the twenties, Einstein found several occasions to publicly address the role of rods and clocks in relativity theory. One of the more famous passages can be found in his delayed lecture for his 1921-22 Nobel Prize, delivered to the Nordic Assembly of Naturalists at Gothenburg in July 1923:

The concept of the rigid body (and that of the clock) has a key bearing on the foregoing consideration of the fundamentals of mechanics, a bearing which there is some justification for challenging. The rigid body is only approximately achieved in Nature, not even with desired approximation; this concept does not therefore strictly satisfy the content requirement [Inhaltsforderung]. ${ }^{21}$ It is also logically unjustifiable to base all physical consideration on the rigid or solid body and then finally reconstruct that body atomically by means of elementary physical laws which in turn have been determined by means of the rigid measuring body [...] It would be logically more correct to begin with the whole of the laws and to apply the 'content-requirement' to this whole first, i.e. to put the unambiguous relation to the world of experience last instead of already fulfilling it in an imperfect form for an artificially isolated part, namely the space-time metric. We are not, however, sufficiently advanced in our knowledge of Nature's elementary laws to adopt this more perfect method without going out of our depth. At the close of our considerations we shall see that in the most recent studies there is an attempt, based on ideas by Levi-Civita, Weyl, and Eddington, to implement that logically purer method (Einstein, 1923d, 2-3; my emphasis).

This well-known passage is consistent with Einstein's stance on this matter in previous years, even though he now makes the point in reference to special relativity. The problem is again

\footnotetext{
${ }^{20}$ The philosopher Joseph Winternitz — son of a colleague of Einstein's in Prague and a friend of Leopold Infeld (who mentioned him simply as 'Joseph' in his Infeld, 1942, cf. Ryckman, 2014, 397, n. 21) -rightly attributes this position to Eddington (cf. Eddington, 1923, 146). Einstein wrote a positive review of Winternitz's book (Einstein, 1924a), which is also a good documentation of his stance toward Kant's philosophy (cf. Hentschel, 1987).

${ }^{21}$ Inhaltsforderung is usually translated as 'stipulation of meaning'. However, the translation obscures the fact that the German expression seems to refer to the conditions that a theory has to satisfy in order to have physical content.
} 
establishing which conditions of the abstract concept of a physical theory (coordinate differences in the special theory) can satisfy the Inhaltsforderung, that is, which condition of their physical meaningfulness. Thus, again, Einstein was concerned with an epistemological issue: whether there are isolated parts of a theory that are directly connected to experience, or whether it is only the entire system of physical laws that is physically meaningful.

The same is of course true for general relativity. Initially, Einstein adopted the stance that one must assume the actual existence of rods whose length "would be the same under all circumstances" (Lorentz, 1923, 390), in order to give physical meaning to the claim that, in general, it is impossible to recover distances measured by rigid rods and clocks from coordinate distances without knowing the quantities $g_{\mu \nu}$, which also play the role of the gravitational field. The reference to the 'logically purer method' of Weyl and Eddington at the end of passage just cited seems to show that, in the context of the attempt "to complete the basis of the general theory of relativity" (Einstein, 1923d, 2), by unifying he gravitational field $\left(g_{\mu v}\right)$ and the other known 'physical' field, the electromagnetic field $\left(\varphi_{v}\right)$, this epistemological attitude had become untenable (for Einstein's conception of unification, cf. Lehmkuhl, 2013). Such a theory (Einstein and Grommer, 1923,2) would account for the existence of the electrically charged constituents of matter, which ultimately enter into the very constitution of rods and clocks, which in turn serve to define the gravitational field.

It is thus clear that if one wants to pursue such a program, a very different conception of the relationship between theory and experience should be used. In his more popular writings from this period, Einstein had an opportunity to address this issue in simpler terms. Some of these passages have already attracted the attention of some interpreters (cf. in particular Howard, 2014), but I will present them as further testimony to Einstein's stance on the relationship between theory and measuring devices.

In a 1924 review of a book by Aldolf Elsbach, a minor Neo-Kantian philosopher (Elsbach, 1924), Einstein distinguishes two different 'standpoints' on the question of the relation between geometry and experience:

The position that one takes on these claims depends on whether one grants reality to the practically-rigid body. If yes, then the concept of the interval corresponds to something experiential. Geometry then contains assertions about possible experiments; it is a physical science that is directly underpinned by experimental testing (standpoint A). If the practicallyrigid measuring body is accorded no reality, then geometry alone contains no assertions about experiences (experiments), but instead only geometry with physical sciences taken together (standpoint B). Until now physics has always availed itself of the simpler standpoint A and, for the most part, is indebted to it for its fruitfulness; physics employs it in all of its measurements. [...] But if one adopts standpoint $B$, which seems overly cautious at the present stage of the development of physics, then geometry alone is not experimentally testable. There are then no geometrical measurements whatsoever [...] Viewed from standpoint B, the choice of geometrical concepts and relations is, indeed, determined only on the grounds of simplicity and instrumental utility. Concerning the metrical determination of space, nothing can then be made out empirically [...] because, on this choice of a standpoint, geometry is not a complete physical conceptual system, but only a part of one such (Einstein, 1924b, 1690-1691; tr. Howard, 2014, 367).

The conflict of these two points of view clearly corresponds to the opposition between the unnamed 'defender of practical geometry' (standpoint A), and that of 'Poincare' (standpoint B), the same opposition that Einstein had introduced in the 1921 lecture. In a brief paper published a year later, "Nichteuklidische Geometrie und Physik" (Einstein, 1925), Einstein added another detail, explicitly attributing 'standpoint B' to Hermann von Helmholtz:

In order to see the matter clearly, one must consistently adopt one of two points of view. In the first, one holds that the 'body' of geometry is realized in principle by rigid bodies in nature, provided that certain conditions are met regarding temperature, mechanical strain, etc.; this is the point of view of the practical physicist. In this case, the 'distance' of geometry agrees with a natural object and thereby all propositions of geometry gain the character of assertions about real bodies. This point of view was especially clearly advocated by Helmholtz, and we can add that without him the formulation of relativity theory would have been practically impossible. In the other point of view, one denies in principle the existence of objects that agree with the fundamental concepts of geometry. Then geometry by itself would include no assertions about objects of reality, only geometry taken together with physics. This point of view, which may be more complete for the systematic representation of a finished physics, was expounded particularly clearly by Poincaré. From this standpoint, the entire content of geometry is conventional; which geometry is preferable depends on how 'simple' physics can be made by using geometry to agree with experience (Einstein, 1925, 253; my emphasis; tr. Pesic, 2007, 161).

As in the 1921 lecture on geometry and experience (using nearly the very same words) Einstein 
insists that the 'Helmholtzian' approach, in which the interval is regarded as measured by rods and clocks, made relativity theory physically possible. Relativity theory is nothing but a theory concerning the 'Lagerungs-Gesetze', the possible placements of infinite many infinitesimal rigid rods (isolated from relative motions, temperature differences or other influences). However, the fiction of the existence of perfect rigid bodies that we had initially assumed as at least in principle possible might turn out to be untenable: special relativity has already put restrictions on the use of rigid bodies of finite dimensions (see also Einstein, 1924c, 86) and the theory of elementary quanta might force us to abandon the notion of rigid bodies in the atomic domain (Einstein, 1925, 253) (if, for instance, rigidity depends on the crystalline structure of the rod, we cannot proceed to indefinitely small rods without losing this structure).

We could then refuse to extend the use of the concept of 'interval' when the initial definition in terms of observable entities fails, e.g., inside of elementary particles. Or, as Einstein was inclined to do, we could try to extend its use to a wider domain by integrating it into a more comprehensive theoretical structure, which could then be compared with experience as a self-supporting totality; e.g., we could claim that non-Euclidean geometry is valid inside of elementary particles, if this claim is part of a consistent unified field theory that correctly reproduces such elementary particles as solutions. Einstein is envisaging here a sort of 'Poincaréan' attitude towards the relationship between geometry and experience. After all, the initial definition of the interval that used rigid bodies (and clocks) was obtained by resorting to certain physical processes described by certain physical laws; strictly speaking, 'geometrical' concepts can only be confronted with reality along with such laws.

Leaving aside Einstein's historical references, it is clear that the commitment to this double standard was one of the peculiar features of Einstein's epistemology. It appears again in the entry for "Space-time" in the 13th edition of the Encyclopedia Britannica form 1926. Here, Einstein introduces a similar opposition between the 'defender of practical geometry' and the more sophisticated position defended by unnamed 'consistent thinkers':

\begin{abstract}
A serious difficulty is encountered in the above represented interpretation of geometry in that the rigid body of experience does not correspond exactly with the geometrical body. In stating this I am thinking less of the fact that there are no absolutely definite marks than that temperature, pressure and other circumstances modify the laws relating to position. It is also to be recollected that the structural constituents of matter (such as atom and electron [...]) assumed by physics are not in principle commensurate with rigid bodies, but that nevertheless the concepts of geometry are applied to them and to their parts. For this reason consistent thinkers have been disinclined to allow real contents of facts [reale Tatsachenbestände] to correspond to geometry alone. They considered it preferable to allow the content of experience [Erfahrungsbestände] to correspond to geometry and physics conjointly. [...] Nevertheless, in the opinion of the author it would not be advisable to give up the first view, from which geometry derives its origin. This connection is essentially founded on the belief that the ideal rigid body is an abstraction that is well rooted in the laws of nature (Einstein, 1926, 609; my emphasis; German in the original).
\end{abstract}

This passage repeats Einstein's characteristic position between the two extremes of the epistemological spectrum. It was legitimate to provisionally adopt the point of view according to which "natural geometry refers to the directly observable specification [Angaben] of rods and clocks" (Pauli, 1926, 273). At the same time it was legitimate to try to apply geometrical concepts thus defined to domains where their initial definition is no longer available. As more consistent thinkers have pointed out, an "experimental test of geometry" (Weyl, 1927, 96) is actually impossible; the individual laws of physics and those of geometry "can only be put to the test as a whole" (Weyl, 1927, 96).

\title{
5. 1930s-1950s. Einstein's Last Reflections on Rods and Clocks
}

In the last twenty years of his life, Einstein showed growing trust in the power of mathematical speculation. However, he did not substantially change his opinion about the role of rods and clocks in relativity theory. In particular, in an often-quoted passage from his "Autobiographical Notes", where he confesses his 'sin' towards his initial treatment of rods and clocks as unstructured entities, Einstein seems to address the very same epistemological concerns that occupied him in the early days of general relativity.

Cornelius Lanczos (who was Einstein's assistant during the period of 1928-29), in popular writings (Lanczos, 1931, 1932) on Einstein's new theory based on distant-parallelism (Einstein, 1928a, b, 1930d; cf. Sauer, 2006), described the physics community after the triumph of quantum mechanics, as divided into two epistemological fronts: (1) the 'positivists', who rejected the very use of differential- 
geometrical methods in physics, since they violate the observability criterion (even the $d s^{2}$ is strictly speaking unobservable since there are no infinitesimally small rods); and (2) the 'metaphysicians', who were convinced that the deep structure of nature could be grasped only by means of speculative mathematical constructions (Lanczos, 1931, 104-105; cf. also Lanczos, 1932, 116). ${ }^{22}$

Several passages from different, mostly popular writings from the turn of the thirties show traces of Einstein once again trying to find a precarious equilibrium between the 'positivistic' justification of relativity theory and the 'metaphysical' one, more in consonance with his works on unified field theory (Lanczos, 1931, 99). ${ }^{23}$ On the one hand, Einstein continued to warn his readers that one should not forget that the general-relativistic field equations can be said to 'hold' in reality since they correctly predict the values of the $g_{\mu v}$, that in turn determine the observed behavior of rods and clocks (Einstein, 1928c, 164-165, 1929b, 1930a,c,b, 1936, 356-357). On the other hand, simultaneously to his work on the unified field theory, he showed increasing confidence in the power of pure mathematical speculation, so that 'internal' criteria (such as simplicity) seem to furnish the only assurance of the correspondence between abstract theory and reality: the field equations are 'correct' because they are the simplest generally-covariant differential equations which the $g_{\mu \nu}$ can satisfy (Einstein, 1929a, 1933, 15, 1936, 369).

\subsection{The Einstein-Swann Correspondence}

Despite Einstein's progressively stronger tendency to consider nature the "realization of the simplest conceivable mathematical ideas" (Einstein, 1933, 15; cf. Norton, 2000; Dongen, 2010), he seems to have maintained his characteristic double-truth doctrine concerning the role of rods and clocks in his theory. A particularly clear articulation of this stance is revealed by Einstein's correspondence with the Anglo-American physicist William Francis Gray Swann, which has recently drawn some attention (cf. Brown, 2005, 120, fn. 19; and in particular Hagar, 2008; cf. also Stachel, 1993). In a letter to Nature, written in September 1941 (but published in December) - referring to the "recent discussion between Sir James Jeans and Sir Arthur Eddington" (Swann, 1941, 692) — Swann explained his non-standard view on length contraction as a quantum-mechanical phenomenon that should be explained dynamically by a Lorentz-invariant theory of matter (Brown, 2005, 119-121).

In his reply, Eddington repeated what he had already explained at length in his Tarner lectures in 1938 (Eddington, 1939), and what I have tried to show in the preceding sections. Relativity theory must refer to quantum mechanics but for a very different reason: "relativity theory has to go outside its own borders to obtain the definition of length, without which it cannot begin" (Eddington, 1939, 76; my emphasis). In order to make the theory empirically meaningful we need to make statements about lengths in a remote star or at a remote epoch, implying that there are physical standards that can be constructed there 'identically' to a corresponding terrestrial standard. This is possible only if the two standards can be constructed from a common specification; for instance, because they stay in definite numerical ratio with some dimensionless pure number, such as $h / m c$, the 'wave-length' of the electron, which appears in the fundamental equations of Dirac's electron theory (cf. Eddington, 1941, 692-693), a constant which Eddington (cf. Eddington, 1933), in contrast to Weyl (cf. Weyl, 1934b), still hoped might be connected with the radius of curvature of the universe.

Swann remained unsatisfied with Eddington's reply and decided to write to Einstein himself in January 1942 (the episode is recounted in detail in Hagar, 2008). Einstein's response, however, seems to confirm Eddington's view. The original German and a translation of the letter can be found in Hagar (2008; 2014, 153-154), but I will provide a relevant excerpt:

In the special theory of relativity measuring rods and clocks (idealized, but in principle conceived as realizable) are treated as independent physical objects, which, linked as they are to the coordinates of the theory [...] [,] are consciously not treated as solutions under the basis of structure laws [Struktur-Gesetzen]. ${ }^{24}$ This is well justified because from the point of view of our experiences, the (in principle) existence of those objects that can serve as measures for

\footnotetext{
${ }^{22}$ The opposition is, of course, rough. Weyl, after the discovery of the 'matter fields' and the possibility of deducing the existence of identical particles from their quantization (Darrigol, 1986, cf. Schweber, 1994, ch. 1), had become very critical of Einstein's "geometrical capers [Luftsprünge]" (Weyl, 1931, 56, cf. Scholz, 2006). He even conquered Pauli's hard-to-win admiration (Pauli to Weyl, 26 August 1929, Pauli, 1979, 518-519) for applying his gauge invariance principle to the PauliDirac theory of the spinning electron (Weyl, 1929a, b). However, Weyl did not embrace a positivistic epistemology (Weyl, 1932, 1934a).

${ }^{23}$ In a letter to Moritz Schlick on 28 November 1930 Einstein explicitly used the expression: "you will be surprised by Einstein the 'metaphysician'. But in this sense every four- and two-legged animal is, de facto, a metaphysician" (AEA, 21603).

${ }^{24}$ With the expression 'structure laws', Einstein understands those laws "that lay claim to a general and strict validity, that is fundamental laws of nature" (Einstein to Bohm, 28 October 1954, AEA 08-050, cit. and trans. in Dongen, 2010, 181).
} 
coordinates appears better justified than any particular structure laws, e.g. Maxwell's equations.

But if one does not introduce rods and clocks as independent objects into the theory, one has to have a structural theory in which a length is fundamental [eine Länge fundamental eingeht], which then leads to the existence of solutions in which that length plays a determinant role, so that a continuous sequence of similar solutions no longer exists [...]. Any theory that has a universal length in its foundation, and because of this produces qualitatively distinct solutions of a certain extension, would do the same with regard to the question under examination here (Einstein to Swann, 24 January 1942, AEA, 20-624; my emphasis; tr. s.m.).

Einstein again insists that, even if initially special relativity relied on the experiential fact that rodand clock-like localized and stable structures exist in nature, in principle one should search for a relativistic field theory of matter that would allow for only a discrete number of solutions, from which rod- and clock-like structure could be constructed. This passage however adds a detail that was not explicit in the other similar passages I have quoted, which, as far I can see, confirms the reading of Einstein's attitude towards rods and clocks that I have suggested. According to Einstein, whatever theory one might use - possibly, but not necessarily a field theory (Stachel, 1986a) — the theory should have a fundamental length scale in its foundation. The existence of particles of constant size and charge (and of more complicated structures such as atoms or crystals) would then be explained by the fact that they stay in the same proportion with such a fundamental length, rather than being postulated from the outset as an unexplained fact. This confirms that the issue at stake was to account for the existence of rods and clocks of the same size in order to assure the reproducibility of the measuring units.

Of course, this was only a program and not a theory. The difficulty of accounting for the existence of matter in a field-theoretical framework had been addressed by Einstein in a brief note published in a rather obscure Argentinian journal a year earlier (Einstein, 1941), and later in a paper written with Pauli (Einstein and Pauli, 1943). Under very general conditions, any attempt to base a unified theory on the Riemann tensor would necessarily involve singularities in particle-like solutions (Einstein, 1944; Einstein and Bargmann, 1944). This result represented, of course, a serious threat to Einstein's ambitions, given his deep conviction that a field theory "which claims to be complete (in contrast e.g. to the pure theory of gravitation)" (Einstein and Straus, 1946), should have rigorous solutions "which are regular in the entire space" (Einstein and Straus, 1946, 737).

\subsection{Einstein's Epistemology in the Schilpp Volume}

The letter to Swann provides further evidence that Einstein's epistemological attitude does not seem to have changed at all from the letter to Dällenbach where we started. The opposition between what can be accepted sub specie temporis, as provisional compromise, and what should be maintained sub specie aeterni, in principle, returned again in an often-quoted passage from Einstein's autobiographic notes written for the Schilpp volume:

One is struck [by the fact] that the theory [of special relativity] [...] introduces two kinds of physical things, i.e., (1) measuring rods and clocks, (2) all other things, e.g., the electromagnetic field, the material point, etc. This, in a certain sense, is inconsistent; strictly speaking measuring rods and clocks would have to be represented as solutions of the basic equations (objects consisting of moving atomic configurations), not, as it were, as theoretically self-sufficient entities. However, the procedure justifies itself because it was clear from the very beginning that the postulates of the theory are not strong enough to deduce from them sufficiently complete equations. If one did not wish to forego a physical interpretation of the co-ordinates in general (something which, in itself would be possible), it was better to permit such inconsistency, with the obligation, however, of eliminating it at a later stage of the theory. But one must not legalize the mentioned $\sin$ so far as to imagine that intervals are physical entities of a special type, intrinsically different from other physical variables ('reducing physics to geometry', etc.) (Einstein, 1949a, 59, my emphasis).

This is the passage that is most often taken as evidence that Einstein himself wanted to explain rod contraction and clock dilation in a 'constructive' way. However, in my opinion, the opposition between constructive and principle theories is not the right framework for understanding the passage, which actually does not differ significantly from the many others that I have quoted so far.

Einstein assumed provisionally " $[\mathrm{t}]$ he existence (in principle) of (ideal, viz., perfect) measuring rods and clocks" (Einstein, 1949a, 59), which, if at rest in an inertial system, always measure the true proper time and proper distance, and thus furnish physical interpretation of the coordinates. This is the condition under which special relativity can acquire the status of a physical theory, "which can be experimentally validated or disproved" (Einstein, 1949a, 57). However, relativity theory cannot 
account for the fortunate circumstance that we happen to live in a world in which such physical systems exist. It would be better if we possessed some special-relativistic theory of matter powerful enough to account for the very existence of 'geometrical' objects (rods and clocks) just like any other 'physical' object. Note that Einstein explicitly claims that this would not mean to 'geometrize' physics, but on the contrary would eliminate the arbitrary difference between 'two types of physical things' the geometrical and non-geometrical ones (cf. Lehmkuhl, 2013).

Another beautiful passage referring to general relativity from Einstein's 'Reply to Criticisms' in the Schilpp volume (Einstein, 1949b, 685) seems to confirm this reading:

Everything finally depends upon the question: Can a spectral line be considered as a measure of a 'proper time' $d s\left(d s^{2}=g_{i k} d x_{i} d x_{k}\right)$ (if one takes into consideration regions of cosmic dimensions)? Is there such a thing as a natural object which incorporates the 'naturalmeasuring-stick' independently of its position in four-dimensional space? The affirmation of this question made the invention of the general theory of relativity psychologically possible; however this supposition is logically not necessary. For the construction of the present theory of relativity the following is essential:

(1) Physical things are described by continuous functions, field-variables of four co-ordinates. As long as the topological connection is preserved, these latter can be freely chosen.

(2) The field-variables are tensor-components; among the tensors is a symmetrical tensor $g_{i k}$

for the description of the gravitational field.

(3) There are physical objects, which (in the macroscopic field) measure the invariant ds.

If (1) and (2) are accepted, (3) is plausible, but not necessary. The construction of mathematical theory rests exclusively upon (1) and (2). For the objects used as tools for measurement do not lead an independent existence alongside of the objects implicated by the field-equations (Einstein, 1949b, 685; my emphasis).

Einstein suggested again (as in 1917) that he started out with the provisional, but plausible assumption that in nature there are physical processes that can be used to measure the invariant $d s$, that will 'normalize' it as the unit interval, that is, that rods and clocks that are not influenced by any gravitational field and always have the same relative length and period exist. However, as Einstein pointed out in a letter to an Australian medicine student, this assumption "could be wrong even though the gravitational field equations are not [brauchen nicht falsch zu sein]" (Einstein to Leonard Champion, 7 March 1951, AEA, 25-481). ${ }^{25}$ In what Einstein called a "complete theory of physics as a totality', which of course does not yet exist, the field equations themselves would account for the existence and behavior of those physical systems that we use as tools of measurement. The distinction between geometrical and non-geometrical objects would disappear, together with the distinction between geometrical and non-geometrical fields, which would be unified into one field (cf. Einstein to Lincoln Barnett, 19 June 1948, AEA, 6-58; cit. and tr. in Lehmkuhl, 2013, 10).

It is precisely the latter scenario that would have important epistemological implications. Einstein insists again that one cannot resort to the 'operational' definition of the $g_{i k}$ outside the 'macroscopic', for instance, inside of elementary particles. The application of this concept to the 'microscopic' should be based on different considerations (Einstein, 1949b, 676), e.g., on the fact that the $g_{i k}$ become part of a more encompassing field theory in which such particles appear as solutions: "In a consistent field theory there is no real definition of the field", Einstein wrote to Besso in 1950: "A priori no bridge to the empirical is given", and "a comparison with the empirically known can only be expected to come from finding exact solutions of the system in which empirically 'known' structures and their interactions are 'reflected"' (Einstein to Besso, 15 April 1950, in Speziali, 1972, 438-439, tr. Stachel, 1986a, 376).

Thus, if one does not want to abandon the 'continuum', the very project of a field-theoretical answer to the problem of matter, one has to face its epistemological implications. Thus, Einstein's plea for a dynamical explanation of rods and clocks should be understood against the background of a general philosophical question of how a theory is related to experimental devices, or by keeping in mind - as he put it more emphatically_ "Pilate's famous question: 'What is truth?" (Einstein, 1949b, 676). It is not a coincidence that Einstein, in discussing Hans Reichenbach's 'meaning = verifiability' criterion, found it helpful to explore a different stance toward the relation between theory and experience by resorting to an imaginary dialogue between 'Helmholtz-Reichenbach' and 'Poincaré'.

(1) 'Helmholtz' claims that "the empirically given solid body realizes the concept of 'distance"" (Einstein, 1949b, 676-677): geometry is verifiable, its propositions might be true or false. (2) 'Poincaré' objects that "the empirically given bodies are not rigid" (Einstein, 1949b, 677) and consequently are not to be used as "the embodiment of geometric intervals" (Einstein, 1949b, 677): geometry is not verifiable, its propositions are neither true nor false. (3) 'Helmholtz' replies that one can

\footnotetext{
${ }^{25}$ I thank Diana Kormos-Buchwald for providing me with the original letter.
} 
realize the notion of a rigid body anyway by taking "thermal volume-dependence, elasticity, electroand magneto-striction, etc., into consideration" (Einstein, 1949b, 677). (4) However, in this way'Poincaré' objects - one has to make use of physical laws that describe the behavior of the rods under this influence. Consequently, "not merely [...] geometry but [...] the entire system of physical laws" (Einstein, 1949b, 676-677) is to be compared with experience (cf. also Einstein, 1954b, 91).

Einstein seems to be repeating here the exact position of his 1921 lecture (and of several other minor texts that I have mentioned above); however, he now more clearly separates the pars destruens of Poincaré's argument from the pars construens, which he famously attributes to an anonymous 'non-Positivist':

\begin{abstract}
Non-Positivist: If, under the stated circumstances, you hold distance to be a legitimate concept, how then is it with your basic principle (meaning = verifiability)? [...] Do you not have to admit that, in your sense of the word, no 'meaning' can be attributed to the individual concepts and assertions of a physical theory at all, and to the entire system only insofar as it makes what is given in experience 'intelligible?' Why do the individual concepts which occur in a theory require any specific justification anyway, if they are only indispensable within the framework of the logical structure of the theory, and the theory only in its entirety validates itself? (Einstein, 1949b, 678; my emphasis).
\end{abstract}

There has been a lot of speculation concerning the identity of the non-Positivist. ${ }^{26}$ However, Einstein himself, or at least his later self when he began to have an increasing faith in the power of mathematics, seems to fit the description. He compared the work of the theorist-no matter "how pure a 'positivist' he may fancy himself" - to that of a "tamed metaphysicist" (Einstein, 1950, 13), who believes that the "logically simple is also the real" (Einstein, 1950, 13). If we recall Lanczos' distinction between positivists and metaphysicians, mentioned above (see section 5.1), one can conjecture that Einstein chose the expression 'non-positivist' to avoid the bolder one of 'metaphysicist' or 'metaphysician' that he used on other occasions.

Whoever the non-positivist might be, it is clear that the question at stake was again whether 'individual statements' of a theory can be directly compared with experience, or whether only the simplicity and rigidity of the 'theory as a whole' are signs of its correspondence to nature (Einstein, $1950,13)$. This is the conceptual backdrop against which the whole discussion about rods and clocks should be understood. In November 1952 (the episode is recounted in Howard, 1990, 13), Paul Oppenheim - a philosopher known most for his work with Carl G. "Peter" Hempel- took Einstein to visit Rudolf Carnap. According to Carnap's recollections, when he reminded him of Otto Neurath's famous ship argument against the positivists, Einstein had a significant reaction: "there is no rock bottom, Neurath's reconstruction of the ship afloat. With that he emphatically agreed" (cit. and tr. in Howard, 1990, 13).

\title{
5.3. The Non-Positivist vs. the Positivist. Weyl vs. Pauli Once Again
}

In popular writings over the same decades (Weyl, 1932, 1934a, 1949a), Weyl defended a very similar epistemological view, in spite of his skepticism toward the project of the unified field theory. In the "Appendix F" of the 1949 English-augmented translation (Weyl, 1949b) of his 1927 philosophical monograph (Weyl, 1927) Weyl articulated his position even more clearly. "The rigid rods and the clocks by which Einstein measures the fundamental quantity $d s^{2}$ ", Weyl claims, are assumed to preserve their lengths and periods "in the last instance because charge $e$ and mass $m$ of the composing elementary particles are preserved" (Weyl, 1949b, 288). However, "[t]he systematic theory [...] proceeds in the opposite direction" (Weyl, 1949b, 288). It starts with the $d s^{2}$ defined implicitly by the theory and then introduces a "primitive field quantity" (Weyl, 1949b, 288) to which the Compton wavelength $\mathrm{h} / \mathrm{mc}$ of the particle stays in constant proportion. The quantity $\mathrm{h} / \mathrm{mc}$ furnishes an absolute length standard, which via the spectral lines of atoms, can be used to 'calibrate' the $d s^{2}$. In this way "[t]he behavior of rods and clocks comes out as a remote consequence of the fully developed theory" (Weyl, 1949b, 288).

As we have seen, at the turn of the 1920s, Weyl (and Eddington) had attempted to identify the field quantity in question with the radius of the curvature of the world in his theory. Weyl (in contrast to Eddington) had completely abandoned this approach, forced by the discovery of the fundamental length standard in quantum field theory (cf. Weyl, 1934b). However, as he points out, this "does not essentially alter the basic relationship just described" (Weyl, 1949b, 288). The question then and now was to explain "the most fundamental features in the nature of the universe" (Weyl, 1949b, 288; my emphasis), the composition of the material world out "of one or a few units, existing in a huge number of completely alike specimens" (Weyl, 1949b, 288; my emphasis), to explain how it happens that the "same particle with its definite charge and mass [...] occurs in the world in a large

\footnotetext{
${ }^{26}$ Pierre Duhem has been the most obvious candidate (cf., e.g., Grünbaum, 1963/1973, 133).
} 
number of copies" (Weyl, 1949b, 288; my emphasis).

Classical physics could only deal with 'preserving quantities', which are highly unstable. Their "initial value may be chosen arbitrarily", and "since perturbation can never entirely be eliminated, deviations are apt to occur in the course of time" (Weyl, 1949b, 288). According to Weyl, the only explanation of the existence of particles of definite charge and mass was to imagine that elementary particles behave like 'adjusting quantities', which are not arbitrary and not perturbable; there must be some mechanism that "enforces a definite value that is independent of past history and hence reasserts itself after any disturbances and any lapse of time as soon as the old conditions are restored" (Weyl, 1949b, 288; cf. Fogel, 2008, sec. 3.5). If Mie's program had been realized, Weyl goes on, then adjustment would have been explained in the framework of classical field physics. The theory would then possess only one, or at most a small number, of static spherically-symmetric solutions of definite size. But Mie's program failed. With the development of relativistic quantum mechanics and quantum electrodynamics, the fields were subjected to a 'second quantization' (the classical field variables become quantum operators), "a process by which one passes indeed from one to an indeterminate number of equal particles" (Weyl, 1949b, 289). In this way, however, "equality is accounted for, yet the particular values of charge and mass remain as unexplained as before" (Weyl, 1949b, 289). To solve this problem one would have to derive from the theory that the fine structure constant has a value nearly equal to $1 / 137$, just as one can derive from Euclidean geometry the value of $\pi$.

Of course, Weyl had no intention of defending his 1918 unified field theory, a theory in which he "no longer believe[s]" (Weyl, 1951, 81; cf. also the addendum to the reprint of Weyl, 1918b; published in Weyl, 1956). However, he still argues that the theory confronted general relativity with a legitimate epistemological question: "the definition" of the metric field with the help of rods and clocks that actually exists can of course "only be regarded as a temporary connection to the experience" (Weyl, 1951, 81). The theory should be able to account for the very behavior of those material systems that are used to calibrate the measuring units: "the relations in which the measurement results which are read off from those bodies stay to the fundamental quantities of the theory must be derived " from the laws of physics" (Weyl, 1951, 81).

It is interesting to notice that the roles in the debate had not changed significantly. In a note added to the 1958 English translation (Pauli, 1958) of his encyclopedia article (Pauli 1921), Pauli insisted again that, when he wrote the original text (see sec. 65 discussed in section 3.4 above), he was already "very doubtful regarding the possibility of explaining the atomism of matter, and particularly of electric charge, with the help of classical concepts of continuous fields alone" (Pauli, 1958, 225, n. 23). Already at that time he "felt rather strongly about the fundamental character of the duality (or, as one says since 1927, complementarity) between the measured field and the test body used as measuring instrument" (Pauli, 1958, 225, n. 23; my emphasis). The surprising reference to Bohr's notion of 'complementarity' shows that Pauli consciously denied the very possibility of applying a single conceptual model to the theory and to the measuring instruments that verify it.

In an address written in the year of his death - on the occasion of what would have been Einstein's 80th birthday-Pauli seems to attribute to this problem the same difficulties that Einstein had encountered in trying to solve the problem of matter in the context of classical field theory. In Einstein's theory "the duality between the field and its means of measurement, although latently present in today's quantum theory of fields, ${ }^{27}$ is conceptually not clearly expressed" (Pauli, 1959, 245; tr. Enz, 1973, 791, my emphasis). Precisely for this reason, "[t]he relation of the applicability of the ordinary space-time concept in the small with the properties of the smallest physical objects, the socalled 'elementary particles' is not disclosed" (Pauli, 1959, 245; ; tr. Enz, 1973, 791). Einstein's project of a unified field theory was then considered physically dubious, due to the same issue that had bothered the nineteen-year-old Pauli (see above, section 2.4). This question does not seem to have lost its importance when Pauli's life was drawing to a close: "Einstein's life", he wrote, "ended with a question [posed] to the science of physics and with a behest for a synthesis to us" (Pauli, 1959, 245; tr. Enz, 1973, 791).

\section{Conclusion}

The entire discussion about the role of rods and clocks in relativity theory seems to revolve around a very simple issue. If the crucial discovery of relativity was that elapsed time, just like traveled distance, is route-dependent, the empirical content of relativity theory depends on the fact that the

\footnotetext{
${ }^{27}$ Bohr and Leon Rosenfeld (Bohr and Rosenfeld, 1933) showed that the measurability of the components of the electromagnetic field by means of point charges "in the sense of electron theory" (Bohr and Rosenfeld, 1933, 358) acquires new complexities in quantum field theory. The measurement of the field values at points of space-time (which is not an operator in a Hilbert space) would require an infinite amount of energy.
} 
units of length and time are not. Following Born, we have tentatively called this assumption 'the principle of the physical identity of the units of measure'. The empirical content of both relativity theories seems to depend entirely on the validity of such a principle, and it was the problem of its validity that forced Einstein to call into question the very relationship between a theory and the material devices that serve to verify it.

It is fascinating that by the time of general relativity's second flowering in the sixties, this very same issue returned in the very same form, raising the very same epistemological concerns. In 1959, John Lighton Synge made a "plea for chronometry" (Synge, 1959), insisting that clocks can determine the metric better than measurements made by measuring rods, customarily employed also in recent textbooks (e.g., Møller, 1952). Space-like separations can be measured indirectly using radar methods (Bondi, 1964). In this way, Synge hoped to "make general relativity more operational than it has been hitherto" (Synge, 1960, 105). Synge attributed to Einstein the claim that physical quantities are defined by the operations used to measure them (Synge, 1958, 7, 1960, 104-105). According to Synge this means that the 'mathematical observation' (MO) of time (time, standard clock, ticking of standard clock) acquires a physical meaning if it can be connected with the 'natural observation' (NO) of time (time atom, emission of wave crests of radiation). Synge does not hide from his readers the difficulties raised by the equation $\mathrm{MO}=\mathrm{NO}$, but he is clearly confident that the equation is realized with sufficient accuracy in the case of time measurements. We have the ability to observe virtually standard clocks, since physical entities (such a vibrating atoms, etc.) exist in nature in which the intrinsic forces far exceed any accelerating forces we can apply.

Which atoms should be chosen as standard clocks? "The answer is that it does not matter, provided we use consistently the same type of atom (all atoms of the same type are regarded as identical) and the same pair of energy levels" (Synge, 1960, 106; my emphasis). In fact, "the only effect of changing from one clock to another is to change the unit of time, the ratio of two units being a universal constant" (Synge, 1960, 107). Synge does not hide the concern that "in the present state of physics it is an impertinence to look too closely into the private life of an atom" (Synge, 1963, 34). However, what Synge called the 'chronometric hypothesis' could at least be regarded as reasonable: "if spectroscopy was a complete chaos (equivalently if spectroscopy did not exist), one would hesitate to speak of a standard clock. But spectroscopy reveals a remarkable order in the behaviour of what we may call 'atomic clocks'” (Synge, 1963, 34).

However, Synge's attitude was clearly regarded as epistemologically unsatisfying by other relativists. Wolfgang Kundt and Banesh Hoffmann (1962), along with Robert Marzke and John A. Wheeler (1964, relying on Marzke, 1959) insisted that it was preferable to eliminate from the process of measurement of the gravitational field all measuring tools whose working mechanism is not described within general relativity. When we use the red-orange spectral line emitted by the krypton-86 isotope as a standard of length (which in 1960 was chosen to replace the Paris platinum-iridium standard), this definition depends on the physical constants which determine the size of that atom, in particular on the fact that they are time-independent (strong equivalence principle). However, the ratio of physical constants, particularly the fine structure constant, may change with time, for instance, as a consequence of the expansion of the universe: "Then the ratio between the two standard meters will also vary. We shall be faced with a serious difficulty. Which meter is right? And, if one is wrong, how do we know that both are not wrong?" (Marzke and Wheeler, 1964, 48).

As Marzke and Wheeler pointed put-a space-time theory "in and by itself" should provide "its own means for defining intervals of space and time", without leaning "at all upon the atomic constitution of matter to define a standard of length" (Marzke and Wheeler, 1964, 62). Marzke's method for the measurements of the proper times between events in space-time involves the use of a so-called 'geodesic clock'. The latter consists of two mirrors traveling along parallel paths in spacetime with a light beam reflecting back and forth between them. The number of reflections yields a well-defined measurement of the space-time interval. Marzke and Wheeler hoped that this standard would "supersede the krypton-86 standard meter as well as the platinum-iridium standard meter by a geometrodynamic standard meter" (Marzke and Wheeler, 1964, 62).

This hope was probably misplaced - the electromagnetic theory of light is scale invariant, so that geodesic clocks cannot be used to set a global definition of a unit of time. However, the epistemological ideal that inspired such attempts is clear. As James Anderson put it, in general, a well-behaved physical theory must satisfy a fundamental requirement: "it must contain a description of all physical systems with which it purports to deal, including those systems employed in the measurement process" (Anderson, 1967, 139; cf. also Anderson, 1964). It is under the spell of this epistemological ideal that several attempts were made to determine the metric structure of space-time, using less structured entities, such as Mario Castagnino (1971), and in particular Jürgen Ehlers, Felix Pirani and Alfred Schild (1972, following the above-mentioned Weyl, 1921b). However, it turned out to be impossible to revert to a full pseudo-Riemannian geometry, with its global definition of the 
measuring units, without avoiding quantum-theoretic considerations (Ehlers, 1973).

Relativists were once again facing the very same problems that the pioneers of the theory had been concerned with: the physical problem of establishing a global definition of the units of measure, and the philosophical issue of establishing to what extent this is possible without abandoning the framework of relativity theory. As Anderson put it with usual clarity, in a conclusion to a paper written for John Stachel's 70th birthday, this is the fundamental epistemological alternative in light of which the whole debate about the role of rods and clocks in relativity theory should be understood:

There are two positions one can take concerning the role of measuring devices in a physical theory. One is that such devices lie outside the province of the theory and their properties must be postulated. Such a view is common in quantum mechanics, in which measuring devices are held to be classical devices, and leads to a number of problems associated with the socalled measurement problem. The other position holds that measuring devices are physical systems whose behavior must be describable within the framework of the theory of the systems they are designed to measure (Anderson, 2003, 280).

As we have seen, Einstein's vacillation between the treatment of clocks as unstructured and structured entities should be interpreted as the attempt to position himself between these alternatives, which are historically well represented by Pauli and Weyl.

Einstein's unease about the 'sin' of black-boxing rods and clocks as simple, rather than treating them as complicated dynamical systems, was precisely an attempt to give a balanced answer to a philosophical question, which he jokingly compared to Pilate's question: 'What is truth?' More humbly, this was the question of whether a theory should describe its own means of verification or whether this description should lie outside its domain. Einstein did not consider this an either-or choice. He became aware that testing a theory is a complex process, which might involve preliminary stages that only provisionally secure the empirical support of the theory, yet be rejected at later stages of its development. Thus, it is only the process as a whole that can be compared with experience in any meaningful way. Writing to his lifelong friend Maurice Solovine in 1953, Einstein remarked that the reduction of geometry to the behavior of rigid bodies is strictly speaking untenable (perfectly rigid bodies do not exist in nature), but at the same time it was inevitable from a didactic or heuristic point of view: "Moral: unless one sins against logic one generally gets nowhere; or one cannot construct a house or a bridge without using a scaffold which is really not one of its basic parts" (Einstein to Solovine, 28 May 1953, AEA, 21-300, tr. in Solovine, 1987, 147).

\section{Acknowledgements}

I thankfully acknowledge the financial support of this work by the DFG (Deutsche Forschungsgemeinschaft). The paper was written while I was a fellow at the Center for Philosophy of Science in Pittsburgh. I would like to express my gratitude to John Norton for many helpful discussions and suggestions. Ori Belkind and Joshua Rosaler made very useful comments on an early draft of the paper. I'm grateful to Dennis Lehmkuhl for an email exchange during the initial stage of writing that turned out to be decisive. I thank Michel Janssen for correcting imprecisions in a previous version of the paper and suggesting several improvements. Any remaining deficiencies in the paper are solely my responsibility.

\footnotetext{
Abbreviations

AEA The Albert Einstein Archives at The Hebrew University of Jerusalem (n.d.).

CPAE Albert Einstein (1987-). The Collected Papers of Albert Einstein. Ed. by John Stachel et al. 13 vols. Princeton: Princeton University Press.
}

\section{References}

Abraham, Max (1905). Theorie der Elektrizität. Vol. 2: Elektromagnetische Theorie der Strahlung. Leipzig: Teubner. Afriat, Alexander (2009). "How Weyl Stumbled Across Electricity while Pursuing Mathematical Justice." In: Studies in History and Philosophy of Science Part B: Studies in History and Philosophy of Modern Physics 40, $20-25$.

Anderson, James L. (1964). "Twins, Clocks and Geometry." In: Transactions of the New York Academy of Sciences 26 (8, Series II), 934-946.

- (1967). Principles of Relativity Physics. New York, NY et al.: Academic Press

- (2003). "Timekeeping in an Expanding Universe." In: Revisiting the Foundations of Relativistic Physics. Festschrift in Honor of John Stachel. Ed. by Jürgen Renn et al. Springer Netherlands, 275-280. 
Barbour, Julian B. (2007). “Einstein and Mach's Principle.” In: The Genesis of General Relativity. Ed. by Jürgen Renn. 4 vols. Dordrecht: Springer, Vol. 4: 569-604.

Bell, John L. and Herbert Korté (2014). Hermann Weyl. Ed. by Edward N. Zalta. url: http://plato.stanford. edu/archives/spr2014/entries/weyl/.

Beller, Mara (1999). Quantum Dialogue. The Making of a Revolution. Chicago, IL: University of Chicago Press.

Blumenthal, Otto, ed. (1913). Das Relativitätsprinzip. Eine Sammlung von Abhandlungen, mit Anmerkungen von A. Sommerfeld und Vorwort von O. Blumenthal. Leipzig/Berlin: Teubner.

Bohr, Niels (1913a). “On the Constitution of Atoms and Molecules. Part I.” In: Philosophical Magazine (26), 1-24.

- (1913b). "On the Constitution of Atoms and Molecules. Part II. Systems Containing Only a Single Nucleus.” In: Philosophical Magazine (26), 476-502.

- (1913c). "On the Constitution of Atoms and Molecules. Part III. Systems Containing Several Nuclei.” In: Philosophical Magazine (26), 857-875.

Bohr, Niels and Leon Rosenfeld (1933). "Zur Frage der Messbarkeit der Elektromagnetischen Feldgrössen.” In: Kgl. Danske Videnskabernes Selskab. Mathematisk-fysiske Meddelelser, 3-65.

Bondi, Hermann (1964). Relativity and Common Sense. A New Approach to Einstein. Garden City, NY: Anchor Books.

Born, Max (1909). "Die Theorie des starren Elektrons in der Kinematik des Relativitätsprinzips.” In: Annalen der Physik $11,1-56$.

- (1915). Dynamik der Kristal lgitter. Leipzig: Teubner.

- (1920). Die Relativitätstheorie Einsteins. Berlin: Springer.

- (1922). Einstein's Theory of Relativity. Trans. by Henry L. Brose. New York, NY: Dutton.

- (1925). "Vorlesungen über Atommechanik. Erster Band." In: Struktur Der Materie in Einzeldarstel lungen. Vol. 2. Berlin: Springer.

Born, Max and Pascual Jordan (1925). “Zur Quantentheorie aperiodischer Vorgänge.” In: Zeitschrift für Physik 33 (1), 479-505.

Brown, Harvey R. (2005). Physical Relativity. Space-Time Structure From a Dynamical Perspective. Oxford: The Clarendon Press.

- (2009). "The Behaviour of Rods and Clocks in General Relativity, and the Meaning of the Metric Field." In: Beyond Einstein: Essays on Geometry, Gravitation, and Cosmology. Ed. by David E. Rowe, Boston, Birkhäuser, 2009

Brown, Harvey R. \& Pooley, Oliver (2006). “Minkowski space-time: A glorious non-entity”. In Dennis Dieks (ed.), The Ontology of Spacetime. Amsterdam et al.: Elsevier. 67--89.

Butterfield, Jeremy (2002). “The End of Time?” In: British Journal for the Philosophy of Science 53, 289-330.

Camilleri, Kristian (2009). Heisenberg and the Interpretation of Quantum Mechanics. The Physicist as Philosopher. Cambridge: Cambridge University Press.

Castagnino, Mario A. (1971). "The Riemannian Structure of Space-Time as a Consequence of a Measurement Method." In: Journal of Mathematical Physics 12 (10), 2203-2211.

Cunningham, Ebenezer (1921). Relativity, Electron Theory and Gravitation. London/New York, NY: Longmans, Green/Co.

Dällenbach, Walter (1918). "Die allgemein kovarianten Grundgleichungen des elektromagnetischen Feldes im Innern ponderabler Materie vom Standpunkt der Elektronentheorie.” PhD thesis. Eidgenössische Technische Hochschule Zürich. Repr. as Dällenbach, 1919.

- (1919). "Die allgemein kovarianten Grundgleichungen des elektromagnetischen Feldes im Innern ponderabler Materie vom Standpunkt der Elektronentheorie." In: Annalen der Physik 363 (6), 523-548.

Darrigol, Olivier (1986). "The Origin of Quantized Matter Waves." In: Historical Studies in the Physical and Biological Sciences 16 (2), 197-253.

Dongen, Jeroen van (2010). Einstein's Unification. Cambridge and New York, NY: Cambridge University Press.

Eddington, Arthur Stanley (1918). Report on the Relativity Theory of Gravitation. 1st ed. London: Fleetway Press.

- (1920a). "The Meaning of Matter and Laws of Nature according to the Theory of Relativity." In: Mind n.s. 29, 145158.

- (1920b). Space, Time and Gravitation. An Outline of the General Relativity Theory. Cambridge: Cambridge University Press.

- (1921a). "A Generalization of Weyl's Theory of the Electromagnetic and Gravitation Fields." In: Proceedings of the Royal Society of London 99, 104-121.

- (1921b). "The Relativity of Field and Matter.” In: Philosophical Magazine 42 (251), 800-806.

- (1922). The Theory of Relativity and its Influence on Scientific thought. Oxford: The Clarendon Press.

- (1923). The Mathematical Theory of Relativity. Cambridge: Cambridge University Press.

- (1933). The Expanding Universe. Cambridge: Cambridge University Press.

- (1939). The Philosophy of Physical Science. Tarner Lectures, 1938. Cambridge: Cambridge University Press.

- (1941). "Philosophy of Physical Science." In: Nature 148, 692-693.

Ehlers, Jürgen (1973). "Survey of General Relativity Theory." In: Relativity, Astrophysics and Cosmology. Proceedings of the Summer School, held 14-26 August, 1972 at the Banff Centre, Banff, Alberta. Ed. by W. Israel. Dordrecht and Boston, MA: Reidel, 1-126.

- (1988). "Weyl, Hermann's Contributions to the General Theory of Relativity." In: Exact Sciences and Their Philosophical Foundations. Vorträge des Internationalen Hermann-Weyl-Kongresses, Kiel 1985 = Exakte Wissenschaften und ihre philosophische Grundlegung. Ed. by Wolfgang Deppert. Frankfurt am Main et al.: Lang.

Ehlers, Jürgen, Felix Arnold Edward Pirani, and Alfred Schild (1972). "The Geometry of Free Fall and Light Propagation." In: General Relativity. Papers in Honour of J. L. Synge. Ed. by Lochlainn O'Raifeartaigh. Oxford: The Clarendon Press, 63-84.

Ehrenfest, Paul (1909). “Gleichförmige Rotation starrer Körper und Relativitätstheorie.” In: Physikalische Zeitschrift 10,918.

Einstein, Albert (1905). "Zur Elektrodynamik bewegter Körper.” In: Annalen der Physik 17, 891-921. Repr. in CPAE, Vol. 2, Doc. 23

— (1906). "Über die Möglichkeit einer neuen Prüfung des Relativitätsprinzips.” In: Annalen der Physik, $1907-198$.

- (1907). "Relativitätsprinzip und die aus demselben gezogenen Folgerungen." In: Jahrbuch der Radioaktivität 4, 411-462. Repr. in CPAE, Vol. 2, Doc. 47.

— (1909a). "Zum gegenwärtigen Stand des Strahlungsproblems.” In: Physikalische Zeitschrift 10, 185-193.

- (1909b). "Über die Entwicklung unserer Anschauungen über das Wesen und die Konstitution der Strahlung." In: Physikalische Zeitschrift 22 (10), 817-826. 
— (1910). "Le principe de relativité et ses conséquences dans la physique moderne." Trans. by Edouard Guillaume. In: Archives des sciences physiques et naturel les 29, 5-28, 125-144. Repr. in CPAE, Vol. 3, Doc. 2.

- (1911a). “Die Relativitäts-Theorie.” In: Naturforschende Gesellschaft in Zürich, Vierteljahresschrift 56, 1-14. Repr. in CPAE, Vol. 3, Doc. 17.

- (1911b). "Diskussion von, 1911a." In: Naturforschende Gesellschaft in Zürich, Vierteljahresschrift 56, II-IX. Repr. in CPAE, Vol. 3, Doc. 18 .

- (1913). "Zum gegenwärtigen Stande des Gravitationsproblems.” In: Physikalische Zeitschrift 14, $1249-1266$. CPAE, Vol. 4, Doc. 17.

- (1916a). "Näherungsweise Integration der Feldgleichungen der Gravitation." In: Sitzungsberichte der Preussischen Akademie der Wissenschaften, 688-696. Physikalisch-mathematische.

- (1916b). "Die Grundlage der allgemeinen Relativitätstheorie.” In: Annalen der Physik 49, 769-822. Also issued as a separatim, Leipzig. J. Barth repr. in CPAE, Vol. 6, Doc 30.

- (1917a). Über die speziel le und die al lgemeine Relativitätstheorie (gemeinverständlich). Braunschweig: Vieweg. Repr. in CPAE, Vol. 6, Doc. 42.

- (1917b). "Kosmologische Betrachtungen zur allgemeinen Relativitätstheorie.” In: Sitzungsberichte der Preussischen Akademie der Wissenschaften, 142-152. Repr. in CPAE, Vol. 6, Doc. 43.

- (1918a). "Review of Weyl, 1918a." In: Die Naturwissenschaften 6, 373. Repr. in CPAE, Vol. 7, Doc. 10.

- (1918b). "Nachtrag zu H. Weyl, Gravitation und Elektrizität [Weyl, 1918b]." In: Sitzungsberichte der Preussischen Akademie der Wissenschaften, 478-80. Repr. in CPAE, Vol. 7, Doc. 8.

- (1919a). "Spielen Gravitationsfelder im Aufbau der materiellen Elementarteilchen eine wesentliche Rolle?" In: Sitzungsberichte der Königlich Preußischen Akademie der Wissenschaften, 349-356. Repr. in CPAE, Vol. 7, Doc. 17

— (1919b). “What is the Theory of Relativity?” In: London Times, 28 November, pages. Repr. in CPAE, Vol. 7, Doc. 25.

- (1920a). “Grundgedanken und Methoden der Relativitätstheorie, in ihrer Entwicklung dargestellt.” First published as CPAE, Vol. 7, Doc. 31.

- (1920b). Äther und Relativitätstheorie. Rede gehalten am 5. Mai 1920 an der Reichs-Universität zu Leiden. Berlin: Springer. Repr. in CPAE, Vol. 7, Doc. 38.

- (1921a). "Geometrie und Erfahrung." In: Sitzungsberichte der Preussischen Akademie der Wissenschaften, 123130. Lecture before the Prussian Academy of Sciences, 27 January 1921. Repr. in expanded form as Geometrie und Erfahrung. Erweiterte Fassung des Festvortrages gehalten an der Preussischen Akademie der Wissenschaften zu Berlin am 27. Januar 1921. Berlin: Springer, 1921. Repr. in CPAE, Vol. 7, Doc. 52.

- (1921b). "Über eine naheliegende Ergänzung des Fundamentes der allgemeinen Relativitätstheorie." In: Sitzungsberichte der Preussischen Akademie der Wissenschaften, 261-264. Repr. in CPAE, Vol. 7, Doc. 54.

- (1921c), "A Brief Outline of the Development of the Theory of Relativity.” In: Nature 1921, 106, 782-784, Repr. in CPAE, Vol. 7, Doc. 53

— (1922a). "Review of Pauli, 1921." In: Die Naturwissenschaften (10), 184-185.

- (1922b). Vier Vorlesungen über Relativitätstheorie, gehalten im Mai 1921, an der Universität Princeton. Braunschweig: Vieweg. Repr. in CPAE, Vol. 7, Doc. 71.

- (1923a). The Meaning of Relativity. Four Lectures Delivered at Princeton University, May, 1921. Trans. by Edwin Plimpton Adams. 5th ed. Princeton, NJ: Princeton University Press.

- (1923b). "Zur allgemeinen Relativitätstheorie." In: Sitzungsberichte der Preussischen Akademie der Wissenschaften, Physikalisch-mathematische Klasse, 32-38, 76-77.

- (1923c). "Zur affinen Feldtheorie." In: Sitzungsberichte der Preussischen Akademie der Wissenschaften, Physikalisch-mathematische Klasse, 137-140.

- (1923d). "Grundgedanken und Probleme der Relativitätstheorie.” In: Les Prix Nobel en 1921-1922. Ed. by Carl Gustaf Santesson. Nobel Foundation. Nobel Prize lecture, delivered before the Nordische Naturforscherversammlung in Göteborg, 11 July, 1923.

— (1923e). "Theory of the Affine Field." In: Nature (112), 448-449.

- (1924a). "Review of Winternitz, 1923." In: Deutsche Literaturzeitung 45, 20-22.

- (1924b). "Review of Elsbach, 1924.” In: Deutsche Literaturzeitung 45, 1685-1692.

— (1924c). "Über den Äther.” In: Verhandlungen der Schweizerischen Naturforschenden Gesellschaft 105, 85-93.

- (1925). "Nichteuklidische Geometrie und Physik." In: Die neue Rundschau 36, 16-20.

- (1926). "Space-time." In: Encyclopedia Britannica. 13th ed. Chicago, IL: Encyclopædia Britannica, Inc., $608-609$.

- (1928a). "Neue Möglichkeit für eine einheitliche Feldtheorie von Gravitation und Elektrizität.” In: Sitzungsberichte der Preussischen Akademie der Wissenschaften, 224-227. Physikalisch-mathematische.

- (1928b). "Riemanngeometrie mit Aufrechterhaltung des Begriffes des Fern-Parallelismus." In: Sitzungsberichte der Preussischen Akademie der Wissenschaften, 217-221. Physikalisch-mathematische Klasse.

- (1928c). "A propos de 'La Deduction Relativiste’ de M. Émile Meyerson.” In: Revue Philosophique de la France Et de L'Étranger (105), 161-66.

— (1929a). "Über den gegenwärtigen Stand der Feldtheorie.” In: Festschrift zum 70. Geburtstag von Prof. Dr. A. Stodola. Ed. by E. Honegger. Zürich: Füssli, 126-132.

- (1929b). "The History of Field Theory (The Olds and News of Field Theory)." In: The New York Times.

- (1930a). "Raum, Äther und Feld in der Physik.” In: Forum Philosophicum 1, 173-180. tr. in the same volume, $180-184$.

- (1930b). "Das Raum-, Feld- und Äther-Problem in der Physik.” In: Koralle 5, 486-487.

- (1930c). "Das Raum-, Feld- und Äther-Problem in der Physik." In: Gesamtbericht, Zweite Weltkraftkonferenz 19, 1-5.

- (1930d). In: Auf die Riemann-Metrik und den Fern-Paral lelismus gegründete einheitliche Feldtheorie 102 (1), 685697.

- (1933). On the Method of Theoretical Physics. The Herbert Spencer Lecture, delivered at Oxford, 10 June 1933. Oxford: The Clarendon Press.

- (1936). "Physik und Realität/Physics and Reality." In: Franklin Institute, Journal 221, 313-347, English translation by J. Picard, 349-382. Also reprinted in Zeitschrift für freie deutsche Forschung, no. 1, 5-19 and no. 2, 1-14 (1938).

- (1941). "Demonstration of the non-existence of gravitational fields with a non-vanishing total mass free of singularities." In: Revista de la Universidad Nacional de Tucuman (A2), 11-16.

— (1944). "Bivector Fields II." In: Annals of Mathematics 45 (1), 15-23.

- (1949a). “Autobiographical Notes.” In: Albert Einstein, Philosopher-Scientist. Ed. by Paul Arthur Schilpp. Evanston, IL: Library of Living Philosophers. 
- (1949b). "Remarks Concerning the Essays Brought together in this Co-operative Volume." In: Albert Einstein, Philosopher-Scientist. Ed. by Paul Arthur Schilpp. Evanston, IL: Library of Living Philosophers.

- (1950). "On the Generalized Theory of Gravitation." In: Scientific American 182, 13-17.

- (1954a). Ideas and Opinions. Ed. by Carl Seelig. New York, NY: Crown Publishers.

- (1954b). "Relativität und Raumproblem." In: Über die speziel le und die al lgemeine Relativitätstheorie (gemeinverständlich). 16th ed. Braunschweig: Vieweg. Appendix 5, added to the 16th edition, Repr. in CPAE, Vol. 6, Doc. 42, 517-534.

Einstein, Albert and Valentine Bargmann (1944). “Bivector Fields.” In: Annals of Mathematics 45 (1), 1--14

Einstein, Albert and Jakob Grommer (1923). "Beweis der Nichtexistenz eines überall regulären zentrisch sym metrischen Feldes nach der Feld-Theorie von Th. Kaluza.” In: Scripta Universitatis atque Bibliothecae Hierosolymitanarum, Mathematica et Physica (Jerusalem) (1), 1-5.

Einstein, Albert and Wolfgang Pauli (1943). "On the Non-Existence of Regular Stationary Solutions of Relativistic Field Equations." In: 44 (2), 131-137.

Einstein, Albert and Maurice Solovine (1987). Letters to Solovine: 1906-1955. New York, NY: Philosophical Library/Open Road.

Einstein, Albert and Ernst G. Straus (1946). “A Generalization of the Relativistic Theory of Gravitation, II.” In: 47 (4),

731-741.

Elsbach, Alfred C. (1924). Kant und Einstein. Untersuchungen über das Verhältnis der modernen Erkenntnistheorie zur Relativitätstheorie. Berlin: de Gruyter.

Charles P. Enz (1973). “W. Pauli's Scientific Work.” In: The Physicist's Conception of Nature. Ed. By Jagdish Mehra. Dordrecht:

Reidel.

Flamm, Ludwig (1916). “Beiträge zur Einstein'schen Gravitationstheorie.” In: Physikalische Zeitschrift 17, 448-454.

Fogel, Brandon (2008). Epistemology of a Theory of Everything: Weyl, Einstein, and the Unification of Physics. Notre Dame, IN: University of Notre Dame, Diss.

Friedman, Michael (2002). “Geometry as a Branch of Physics: Background and Context for Einstein's 'Geometry and Experience'." In: Reading Natural Philosophy. Ed. by David Malament. Chicago-La Salle (IL): Open Court.

- (2010). "Synthetic History Reconsidered." In: Discourse on a New Method: Reinvigorating the Marriage of History and Philosophy of Science. Ed. by Michael Dickson and Mary Domski. Chicago, IL: Open Court.

- (2014). "Space, Time, and Geometry." In: The Cambridge Companion to Einstein. Ed. by Michel Janssen and Christoph Lehner. Cambridge: Cambridge University Press, 398-420.

Gorski, Matthew (2010). Brown and Janssen on the Arrow of Explanation in Special Relativity. url: http: //philsci-archive.pitt.edu/8390/.

Grebe, Leonhard (1920). "Über die Gravitationsverschiebung der Fraunhoferschen Linien." In: Physikalische Zeitschrift 21, 662-668.

Grünbaum, Adolf (1963/1973). Philosophical Problems of Space and Time. Dordrecht, Boston, MA/London: Reidel.

Hagar, Amit (2008). "Length Matters: The Einstein-Swann Correspondence and the Constructive Approach to the Special Theory of Relativity." In: Studies in History and Philosophy of Science Part B: Studies in History and Philosophy of Modern Physics 39 (3), 532-556.

- (2014). Discrete or Continuous? The Quest for Fundamental Length in Modern Physics. Cambridge: Cambridge University Press.

Heisenberg, Werner (1925). "Über quantentheoretische Umdeutung kinematischer und mechanischer Beziehungen.” In: Zeitschrift fur Physik 33, 879-893.

- (1966). Der Teil und das Ganze. Gespräche im Umkreis der Atomphysik. München: R. Piper \& Co. Verlag.

Hemmo, Meir and Amit Hagar (2013). "The Primacy of Geometry.” In: Studies in the History and Philosophy of

Science. Part B: Studies in the History and Philosophy of Modern Physics, 357-364.

Hendry, John (1984). The Creation of Quantum Mechanics and the Bohr-Pauli Dialogue. Dordrecht: Reidel.

Hentschel, Klaus (1987). "Einstein, Neokantianismus und Theorienholismus.” In: Kant-Studien 78, 459-470.

- (1992a). "Einstein's Attitude Towards Experiments: Testing Relativity Theory 1907-1927." In: Studies in

History and Philosophy of Science 23 (4), 593-624.

- (1992b). "Grebe/Bachems photometrische Analyse der Linienprofile und die Gravitations-Rotverschiebung:

1919 bis 1922.” In: Annals of Science 49 (1), 21-46.

- (1994). "Erwin Finlay Freundlich and Testing Einstein's Theory of Relativity." In: Archive for the History of the Exact Sciences 47, 143-201.

Herglotz, Gustav (1910). “Über den vom Standpunkt des Relativitätsprinzips aus als 'starr' zu bezeichnenden Körper.” In: Annalen der Physik 31, 393-415.

Hilbert, David (1915). “Grundlagen der Physik, Erste Mitteilung, vorgelegt in der Sitzung vom 20. November 1915." In: Nachrichten von der Königl. Gesellschaft der Wissenschaften und der Universität zu Göttingenn

Math-physik. Klasse, 1915 395-407. Repr. Hilbert, 2009, ch. I, 28-46.

- (1916/1917). "Die Grundlagen der Physik II.” Georg-August-Universität Göttingen, Mathematisches Institut, Lesesaal. Repr. in Hilbert 2009, 162-307.

- (2009). David Hilbert's Lectures on the Foundations of Physics 1915-1927., Ed. by Tilman Sauer and Ulrich Majer. Berlin/Heidelberg: Springer.

Howard, Don (1990). “Einstein and Duhem.” In: Synthese 83 (3), 363-384.

- (1994). "Einstein, Kant and the Origins of Logical Empiricism." In: Logic, Language, and the Structure of Scientific theories. Proceedings of the Carnap-Reichenbach Centennial, University of Konstanz, 21-24 May 1991. Ed. by Wesley C. Salmon and Gereon Wolters. Pittsburgh, PA/Konstanz: University of Pittsburgh Press/Universitätsverlag Konstanz.

- (2005). Einstein's Philosophy of Science. Ed. by Edward N. Zalta. url: http://plato.stanford.edu/entries/ einstein-philscience/.

- (2007). “'And I Shall not Mingle Conjectures with Certainties'. On the Intellectual Background to Einstein's Distinction between Principle Theories and Constructive Theories." Manuscript.

- (2014). "Einstein and the Development of Twentieth-Century Philosophy of Science." In: The Cambridge Companion to Einstein. Ed. by Michel Janssen and Christoph Lehner. Cambridge: Cambridge University Press, 354-376.

Infeld, Leopold (1942). Quest. The Evolution of a Scientist. London: Readers Union.

Janssen, Michel (2009). "Drawing the line between Kinematics and Dynamics in Special Relativity." In: Studies in History and Philosophy of Science Part B: Studies in History and Philosophy of Modern Physics 40 (1), 26-52.

Janssen, Michel and Christoph Lehner, eds. (2014). The Cambridge Companion to Einstein. Cambridge: Cambridge 
University Press.

Kretschmann, Erich (1918). "Über den physikalischen Sinn der Relativitätspostulate. A. Einsteins neue und seine ursprüngliche Relativitätstheorie.” In: Annalen der Physik 53, 575-614.

Kundt, Wolfgang and Banesh Hoffman (1962). "Determination of Gravitational Standard Time.” In: Recent Developments in General Relativity. New York, NY: Pergamon.

Lanczos, Cornel (1931). "Die neue Feldtheorie Einsteins." In: Ergebnisse der exakten Naturwissenschaften 10, $97-$ 132.

- (1932). "Stellung der Relativitätstheorie zu anderen physikalischen Theorien.” In: Die Naturwissenschaften 20 (7), $113-116$.

Laue, Max von (1911). “Zur Diskussion über den starren Körper in der Relativitätstheorie.” In: Physikalische Zeitschrift 12, 85-87.

- (1920). "Theoretisches über neuere optische Beobachtungen zur Relativitätstheorie." In: Physikalische Zeitschrift 21, 659-662.

Laue, Max von, Walter Friedrich, and Paul Knipping (1913). "Interferenzerscheinungen bei Röntgenstrahlen." In: Annalen der Physik 346 (10), 971-988.

Lehmkuhl, Dennis (2013). Why Einstein did not Believe that General Relativity Geometrizes Gravity. url: http://philsci-archive.pitt.edu/9825/.

Levi-Civita, Tullio (1916). "Nozione di parallelismo in una varietà qualunque e conseguente specificazione geometrica della curvatura riemanniana." In: Rendiconti del Circolo Matematico di Palermo (1884 - 1940) 42 (1), 173-204.

Lorentz, Hendrik Antoon (1910). "Alte und neue Fragen der Physik.” In: Physikalische Zeitschrift (11), 1234-1257.

- (1923). "The Determination of the Potentials in the General Theory of Relativity, with Some Remarks about the Measurement of Lengths and Intervals of Time and about the Theories of Weyl and Eddington." In: Proc. Royal Acad. Amsterdam 29,383-399.

Maltese, Giulio and Lucia Orlando (1995). "The Definition of Rigidity in the Special Theory of Relativity and the Genesis of the General Theory of Relativity." In: Studies In History and Philosophy of Science Part B. Studies In History and Philosophy of Modern Physics 26 (3), 263-306.

Marzke, Robert F. (1959). The Theory of Measurement in General Relativity. Princeton, NJ: Department of Physics. Senior Thesis.

Marzke, Robert F. and John Archibald Wheeler (1964). "Gravitation as Geometry. I. The Geometry of Spacetime and the Geometrodynamical Standard Meter." In: Gravitation and Relativity. Ed. by H.Y. Chiu and W.F. Hoffman. New York, NY: Benjamin.

McCormmach, Russell (1970). "Einstein, Lorentz, and the Electron Theory." In: Historical Studies in the Physical Sciences 2, 41-87.

Mehra, Jagdish and Helmut Rechenberg (1982). The Historical Development of Quantum Theory. Vol. 1, Part 2. New York, NY: Springer.

Mie, Gustav (1912a). "Grundlagen einer Theorie der Materie.” In: Annalen der Physik 37, 511-534.

— (1912b). "Grundlagen einer Theorie der Materie. Zweite Mitteilung." In: Annalen der Physik (39), 1-40.

— (1913). "Grundlagen einer Theorie der Materie. Dritte Mitteilung." In: Annalen der Physik (40), 1-66.

- (1920). "Das elektrische Feld eines um ein Gravitationszentrum rotierenden geladenen Partikelchens." In: Physikalische Zeitschrift 21 (23/24), 651-659. Repr. in Weyl, 1968, 2, Doc. 40. Møller, Christian (1952). The Theory of Relativity. Oxford: The Clarendon Press.

Noether, Fritz (1910). "Zur Kinematik des starren Körpers in der Relativitätstheorie.” In: Annalen der Physik 31, 919944.

Norton, John (2000). “Nature is the Realisation of the Simplest Conceivable Mathematical Ideas'. Einstein and the Canon of Mathematical Simplicity." In: Studies in History and Philosophy of Science Part B: Studies in History and Philosophy of Modern Physics 31 (2), 135-170.

— (2008). "Why Constructive Relativity Fails." In: The British Journal for the Philosophy of Science 59 (4), 821834.

O’Raifeartaigh, L. (1997). The Dawning of Gauge Theory. Princeton, NJ: Princeton University Press.

Pauli, Wolfgang (1919a). "Merkurperihelbewegung und Strahlenablenkung in Weyls Gravitationstheorie." In: Verhandlungen der Deutschen Physikalischen Gesellschaft 21 (21-22), 742-750.

— (1919b). "Über die Energiekomponenten des Gravitationsfeldes." In: Physikalische Zeitschrift 20 (1), 25-27.

- (1919c). "Zur Theorie der Gravitation und der Elektrizität von Hermann Weyl." In: Physikalische Zeitschrift 20 (20), $457-467$.

— (1921). "Relativitätstheorie.” In: Enzyklopädie der mathematischen Wissenschaften. Ed. by Arnold Sommerfeld. Vol. 5: Physik, Part 2. Teubner, 539-775. Repr. in book form as Relativitätstheorie. Leipzig: Teubner, 1921.

— (1926). "Review of Eddington, 1923." In: Die Naturwissenschaften, 273-274.

- (1958). Theory of Relativity. London: Pergamon Press.

— (1959). "Albert Einstein in der Entwicklung der Physik." In: Physikalische Blätter 15 (6), 241-245.

- (1979). Wissenschaftlicher Briefwechsel mit Bohr, Einstein, Heisenberg u. a., Ed. by Karl von Meyenn. Vol. 1: 1919 1929. Berlin: Springer.

Peres, Asher and Wojciech H. Zurek (1982). "Is Quantum Theory Universally Valid?” In: American Journal of Physics 50.9, 807-810.

Pesic, Peter, ed. (2007). Beyond Geometry: Classic Papers from Riemann to Einstein. Mineola, NY: Dover Publications.

Pierseaux, Yves (1999). La structure fine de la théorie de la relativité restreinte. Paris/Montréal (Québec): l'Harmattan

- (2003). "The Principle of Physical Identity of Units of Measure in Einstein's Special Relativity." In: Physica Scripta 68, C59.

Poincaré, Henri (1906). "Sur la dynamique de l'électron.” In: Rendiconti del circolo matematico di Palermo 21, 29-176.

Ryckman, Thomas (2005). The Reign of Relativity. Philosophy in Physics 1915-1925. Oxford/New York, NY: Oxford University Press.

- (2014). “'A believing rationalist' Einstein and 'the truly valuable' in Kant.” In: The Cambridge Companion to Einstein. Ed. by Michel Janssen and Christoph Lehner. Cambridge: Cambridge University Press, 377-397.

Sauer, Tilman (2006). "Field Equations in Teleparallel Space-Time. Einstein's Fernparallelismus Approach toward Unified Field Theory." In: Historia Mathematica 33 (4), 399-439.

Scholz, Erhard (1994). "Weyl, Hermann's Contributions to Geometry, 1917-1923." In: The Intersection of History and Mathematics. Ed. by Saski Chikara, Sugiura Mitsuo, and Joseph W. Dauben. Basel: Birkhäuser, 203-229.

- (2006). "The Changing Concept of Matter in H. Weyl's Thought, 1918-1930." In: Interactions. Mathematics, Physics 
and Philosophy, 1860-1930. Ed. by Vincent F. Hendricks et al. Vol. 251. Springer Netherlands, 281-306.

- (2007). “A. Einstein and H. Weyl: Intertwining Paths and Mutual Influences.” In: Albert Einstein et Hermann Weyl, 1955-2005. Questions épistémologiques ouvertes. Mandurai: Barbieri. Ed. by C. Alunni et al. Mandurai: Barbieri, 215-230.

Schweber, Silvan S. (1994). QED and the Men Who Made it: Dyson, Feynman, Schwinger, and Tomonaga. Princeton, NJ: Princeton University Press

Seelig, Carl (1960). Albert Einstein: Leben und Werk eines Genies unserer Zeit. Vienna: Europa Verlag.

Silberstein, Ludwik (1922). The Theory of General Relativity and Gravitation. Toronto: University of Toronto Press. Based on a course of lectures delivered at the Conference on Recent Advances in Physics held at the University of Toronto, in January, 1921.

Speziali, Pierre, ed. (1972). Albert Einstein-Michele Besso: Correspondance 1903-1955. Paris: Hermann.

St. John, Charles Edward (1917). "The Principle of Generalized Relativity and the Displacement of Fraunhofer Lines Toward the Red.” In: Astrophysical Journal 46, 249.

Stachel, John (1986a). "Einstein and the Quantum. Fifty Years of Struggle.” In: From Quarks to Quasars. Philosophical Problems of Modern Physics. Ed. by Robert Garland Colodny and Alberto. Coffa. Pittsburgh: University of Pittsburgh Press, 349-81. Repr. in Stachel, 2002, 367-402.

- (1986b). "Eddington and Einstein.” In: The Prism of Science. Ed. by Edna Ullmann-Margalit. Vol. 95. Springer Netherlands, 225-250. Repr. in Stachel, 2002, 453-475.

- (1989). "The Rigidly Rotating Disk as the 'Missing Link in the History of General Relativity." In: Einstein and the History of General Relativity. Ed. by Don Howard and John Stachel. Boston, MA: Birkhäuser, 48-62. Repr. in Stachel, 2002, 245-260.

— (1993). “The Other Einstein: Einstein Contra Field Theory.” In: Science in Context 6 (01), 275-290. Repr. In Stachel, 2002, 141-154.

- (2002). Einstein from ' $B$ ' to ' $Z$ '. Boston, MA: Birkhäuser.

- (2005). "Einstein et 'Zweistein'." In: Revue de Synthèse 126 (2), 353-365.

Swann, William Francis Gray (1941). "Philosophy of the Physical Science.” In: Nature (108), 692.

Synge, John Lighton (1958). Relativity. The Special Theory. Amsterdam/New York, NY: North-Holland.

- (1959). “A Plea for Chronometry." In: New Scientist 5 (118), 410-412.

- (1960). Relativity. The General Theory. Amsterdam/New York, NY: North-Holland/Interscience.

- (1963). "Introduction to General Relativity." In: Relativité, groupes et topologie. Relativity, Groups and Topology. Lectures Delivered at Les Houches during the 1963 Session of the Summer School of Theoretical Physics, University of Grenoble. Ed. by Cécile DeWitt-Morette and Bryce S. DeWitt. New York, NY: Gordon/ Breach, 1-88.

Bacelar Valente, Mario (2013). Time in the Theory of Relativity: on Natural Clocks, Proper Time, the Clock Hypothesis, and all That. url: http://philsci-archive.pitt.edu/9819/

Valenti, Antony (2008). "Hidden Variables and the Large-Scale Structure of Spacetime." In: Einstein, Relativity and Absolute Simultaneity, Ed. by William Lane Craig, London: Routledge, 2008

Vizgin, Vladimir Pavlovich (1994). Unified Field Theories in the First Third of the 20th Century. Trans. by Julian Barbour. Boston, MA and Basel/Stuttgart: Birkhäuser.

Weyl, Hermann (1917). “Zur Gravitationstheorie.” In: Annalen der Physik 359 (18), 117-145.

- (1918a). Raum, Zeit, Materie. Vorlesungen über al lgemeine Relativitätstheorie. Berlin: Springer.

- (1918b). "Gravitation und Elektrizität." In: Sitzungsberichte der Preussischen Akademie der Wissenschaften, 465480. Repr. in Weyl, 1968, 2, Doc. 31.

- (1918c). "Erwiderung auf Einsteins Nachtrag zu H. Weyl, Gravitation und Elektrizität [Einstein, 1918b]." In: Sitzungsberichte der Preussischen Akademie der Wissenschaften, 478-80. Repr. in Weyl, 1968, 2, Doc. 31.

— (1918d). "Reine Infinitesimalgeometrie." In: Mathematische Zeitschrift 2 (3), 384-411. Repr. in Weyl, 1968,2 , Doc. 30.

- (1919a). "Eine neue Erweiterung der Relativitätstheorie.” In: Annalen der Physik 59, 101-133. Repr. in Weyl, 1968, 2, Doc. 34.

- (1919b). Raum, Zeit, Materie. Vorlesungen über al lgemeine Relativitätstheorie. 3rd ed. Berlin: Springer.

- (1920). “Elektrizität und Gravitation.” In: Physikalische Zeitschrift 21 (21), 649-650. Repr. in Weyl, 1968,2 , Doc. 40.

- (1921a). Raum, Zeit, Materie. Vorlesungen über al lgemeine Relativitätstheorie. 4th ed. Berlin: Springer.

- (1921b). "Zur Infinitesimalgeometrie. Einordnung der projektiven und konformen Auffassung." In: Nachrichten der Königlichen Gesellschaft der Wissenschaften zu Göttingen. Mathematisch Physikalische Klasse, 99-112. Repr. in Weyl, 1968, 2, Doc. 43.

— (1921c). "Feld und Materie." In: Annalen der Physik 65, 541-563. Repr. in Weyl, 1968, 2, Doc. 47.

— (1921d). "Über die physikalischen Grundlagen der erweiterten Relativitätstheorie.” In: Physikalische Zeitschrift 22, 473-480. Repr. in Weyl, 1968, 2, Doc. 46.

- (1922a). Space-Time-Matter. Ed. by Henry L. Brose. London: Methuen \& Co. Ltd.

- (1922b). "Die Relativitätstheorie auf der Naturforscherversammlung." In: Jahresbericht der Deutschen Mathematikervereinigung 31, 51-63. Repr. in Weyl, 1968, 2, Doc. 52.

- (1923). Raum, Zeit, Materie. Vorlesungen über al lgemeine Relativitätstheorie. 5th ed. Berlin: Springer.

- (1927). "Philosophie der Mathematik und Naturwissenschaft." In: Handbuch der Philosophie. Ed. by Alfred Bäumler and Manfred Schröter. München/Berlin: Oldenbourg.

- (1929a). "Gravitation and the Electron." In: Proceedings of the National Academy of Sciences of the United States of America 15, 323-334. Repr. in Weyl, 1968, 3, Doc. 84.

— (1929b). "Elektron und Gravitation.” In: Zeitschrift für Physik 56, 330-352. Repr. in Weyl, 1968, 3, Doc. 85.

- (1931). "Geometrie und Physik." In: Die Naturwissenschaften 19, 49-58. Repr. in Weyl, 1968, 3, Doc. 93.

- (1932). The Open World: Three Lectures on the Metaphysical Implications of Science. New Haven, CT/London: Yale University Press. H. Milford, Oxford University Press. Repr. in Weyl, 2009, Ch. 4.

- (1934a). Mind and Nature. Philadelphia, PA: University of Pennsylvania Press. Repr. in Weyl, 2009, Ch. 5.

- (1934b). "Universum und Atom.” In: Naturwissenschaften 22 (10), 145-149.

- (1946). "Address at the Princeton Bicentennial Conference." Two versions of the address are preserved in the Weyl's Nachlass at the ETH in Zurich: 1. (Hs 91a:17) "Dec. 1946. Bicentennial conference. First version [not used]"; 2. (Hs 91a:18) "(Bicentennial conference). The manuscript is transcribed in Weyl, 2009, ch. 6. See p. 235 for more details on the manuscript.

- (1949a). "Wissenschaft als symbolische Konstruktion des Menschen.” In: Eranos-Jahrbuch (1948), 375-431 Repr. in Weyl, 1968, 1V, Doc. 142.

— (1949b). Philosophy of Mathematics and Natural Science. Princeton, NJ: Princeton University Press. 
— (1951). "50 Jahre Relativitätstheorie.” In: Die Naturwissenschaften 38 (4), 73-83. Repr. in Weyl, 1968, 3, Doc. 149.

- (1955). "Erkenntnis und Besinnung (Ein Lebensrückblick)." In: Studia Philosophica, 153-171. A talk given at the University of Lausanne, May 1954.

- (1956). Selecta. Herausgegeben zu seinem siebzigsten Geburtstag von der Eidgenössischen Technischen

Hochschule in Zürich und vom Institute for Advanced Study in Princeton. Basel: Birkhäuser.

- (1968). Gesammelte Abhandlungen. Ed. by Komaravolu Chandrasekharan. 4 vols. Berlin: Springer.

- (2009). Mind and Nature. Selected Writings on Philosophy, Mathematics, and Physics. Ed. by Peter Pesic.

Princeton, NJ: Princeton University Press.

Winternitz, Josef (1923). Relativitätstheorie und Erkenntnislehre. Eine Untersuchung über die erkenntnistheoretischen Grundlagen der Einsteinschen Theorie und die Bedeutung ihrer Ergebnisse für die allgemeinen Probleme des Naturerkennens. Leipzig: Teubner. 